\title{
Effect of polar solvents on the crystalline phase of polyamides
}

M. Laurati ${ }^{1,2}$, A. Arbe ${ }^{2}$, A. Rios de Anda ${ }^{1}$, L.-A. Fillot ${ }^{1}$, P. Sotta ${ }^{1}$

1 Laboratoire Polymères et Matériaux Avancés, UMR5268,

CNRS/Solvay Advanced Research \& Technology Innovation,

85 Rue des frères Perret,

69192 Saint-Fons Cedex, France.

${ }^{2}$ Centro de Física de Materiales (CSIC-UPV/EHU),

Paseo Manuel de Lardizabal 5, 20018 Donostia/San Sebastián, Spain. 


\begin{abstract}
We investigate the effect of absorption of polar solvents (water and ethanol) on the structure of amorphous and semi-crystalline copolyamides containing aromatic entities. Different length scales of structural organisation are probed using a combination of Small angle X-ray scattering and neutron/X-rays diffraction. Crystallisation in the presence of solvent, characterised by very slow kinetics, is observed in the originally amorphous material, as a combined effect of a rearrangement of the hydrogen-bond distribution and plasticization. The steady-state crystalline structure is reached on a timescale much longer than the absorption kinetics, and is similar but not identical to that of processed semi-crystalline materials. In semi-crystalline polyamides water absorption induces a swelling of the spacing of the lamellar stacking but also modifications of the unit cell structure, which suggest a moderate but significant rearrangement of the hydrogen bond structure in the crystalline phase.
\end{abstract}

PACS numbers: 62.20.-x,62.10.+s,64.70.pv 


\section{INTRODUCTION}

Polyamides (PAs), also called Nylons, are a class of semi-crystalline polymers which contain amide groups intercalated along linear alkane chains [1-3]. These polymers present pronounced chain-chain interactions, originating from the hydrogen bonding between amide groups of neighbouring chains. Thus, they provide a versatile model for polymers with a relatively high density of hydrogen bonds. The presence of hydrogen bonds plays a major role in the properties of polyamides [4]. For instance in the crystalline phase, different organisation of the hydrogen bonds between neighbour chains is responsible for the observed crystalline polymorphism [5-10].

Interaction with polar solvents affects amide-amide hydrogen bonding. Studies on water absorption in semicrystalline polyamides [11-18] show that water enters preferentially the amorphous phase [19, 20], with a strong effect on its molecular mobility at room temperature and above [14, 21-23]. This plasticisation effect has been documented using different experimental techniques, like NMR [11], dynamic mechanical analysis $[12,13]$ and dielectric spectroscopy $[14,16-18]$. A proposed explanation for plasticisation is that water molecules break the amide-amide bonds and intercalate between amide groups of neighbour chains forming a bridge between them which allows a higher mobility [13]. The solvent-induced plasticisation has a significant impact e.g. on mechanical properties [1]. Most relevant for applications is the long term stability of material properties, which is known to be affected by humidity. It has been shown in addition that water absorption may not be homogeneous throughout the whole amorphous phase, due to different degrees of constraint/confinement within the amorphous phase [24-26]. While most of the water is absorbed in the bulk amorphous phase, some water is absorbed into the amorphous phase comprised in between the 
lamellae of the crystalline phase [20, 24, 27, 28], inducing swelling of the lamellar stacking.

In addition to the effect of swelling of the lamellar phase, there is a limited number of experimental indications that absorption of water could also directly influence the crystalline phase at the level of the unit cell. Murthy and coworkers have shown that water absorption affects the positions of the crystalline $(002)+(202) \alpha$ phase Bragg reflections observed in annealed PA6 [27]. A still open question is whether these effects on the unit cell crystalline organisation are due to water penetration into the crystallites and the induced reorganisation of its hydrogen bond structure, or to an indirect effect of an increased mobility of the amorphous phase.

Furthermore it was recently shown that formation of amide-amide hydrogen bonds can be hindered at high temperature by the strong solvation of amide groups by water molecules, inhibiting crystallisation and/or allowing dissolution of crystallites, as observed in waterimmersed Nylon- 4,6 at a temperature of about $200^{\circ} \mathrm{C}$ [29-31]. The mobility of hydrogen bonds induced in the crystalline phase at high temperature by superheated water was also discussed in analogy with the Brill transition, which takes place in PA6 and consists in reorientations of hydrogen bonds within crystallites [32]. Amide group interactions may be efficiently shielded by aqueous ionic solutions as well [31, 33, 34]. These results suggest that even at room temperature water and other polar solvents might have an effect on the crystallisation of PAs.

In this work we complement the existing investigations of the effects of polar solvents on the hydrogen bond structure of polyamides by comparing directly the structural changes induced by absorption of water and ethanol on two model copolymers, one fully amorphous (PA6,6/6I) and one semi-crystalline (PA6,6/6T), which present a comparable density of amide groups in the amorphous phase, $\mathrm{P}$ comparable number of sites available for forma- 
tion of hydrogen bonds with solvent molecules. For comparison, a similar study is performed on standard aliphatic PA6,6. We investigate in this way the structural reorganisation of the hydrogen bonds in the amorphous and crystalline phases, induced by solvent absorntion at

room temperature. Phe initially fully amorphous copolymer hydrogen bond reorganisaton results in a slow solvent mediated crystallisation which cannot be explained only in terms of plasticisation. In the already semi-crystalline materials the structure at the unit cell level changes in the presence of solvent, indicating that the hydrogen bonds in the crystallites may rearrange under the effect of water.

Furthermore, we compare the kinetics of structural changes and those of absorption for the three polymers: This comparison reveals that crystallisation in the originally amorphous polymer occurs after solvent absorption, while the modifications in the semi-crystalline polymers are observed during the absorption process. The structural changes are 2 served simultaneously over length scales which span from those characteristic of the unit cell to those at which the lamellar stacking is observed, using a combination of scattering measurements in the wide and small angle regimes.

\section{CRYSTALLINE ORGANISATION IN POLYAMIDES}

The most commonly encountered crystalline structure in PA6,6 is the so-called $\alpha$-phase, already described by Bunn and Garner in 1947 [35]. In this phase, hydrogen-bonds are arranged in planes, forming sheets of parallel chains (Figure 1). The crystal then consists of a packing of these sheets through van der Waals interactions. Along a chain, successive amide groups are antiparallel. In PA6,6 processed by melt crystallization, only $\alpha$-crystals are formed, but the structure can vary, according to the thermal history of the material, i. e. to the processing parameters. Crystalline structure parameters can vary continuously between 
(a)

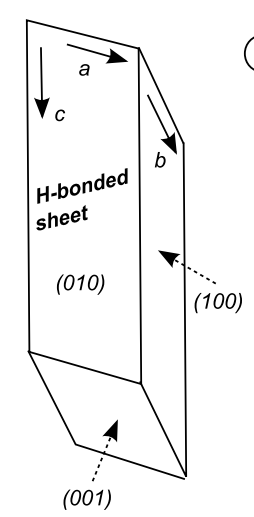

(b)

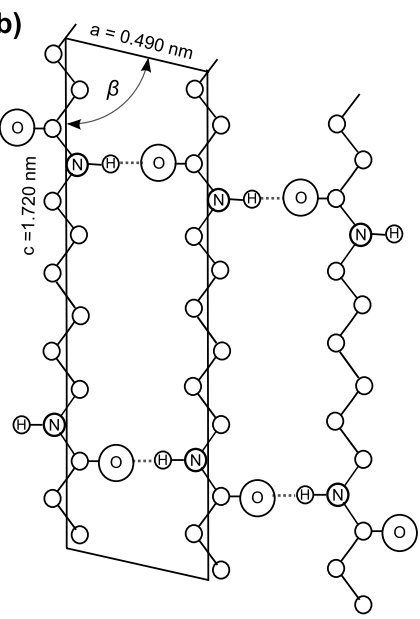

(c)

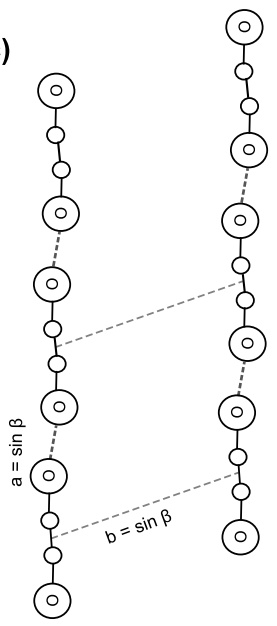

FIG. 1: Schematics of the $\alpha$ phase crystalline structure in PA6,6. (a): triclinic unit cell with base vectors; (b): chain arrangement within the a-c planes, which forms the H-bonded sheets; (c): chain arrangement in plane perpendicular to chain axis. Redrawn from [1].

two limiting forms called $\alpha_{I}$ and $\alpha_{I I}$ : The $\alpha_{I}$ form, generally considered to be the most stable one, is obtained in solution crystallization [36]. The unit cell is triclinic, with a high density $\left(\rho=1.21\right.$ to $\left.1.22 \mathrm{~g} \cdot \mathrm{cm}^{-3}\right)$, and lattice parameters $\left(24^{\circ} \mathrm{C}\right) a=4.97 \AA, b=5.47 \AA$ and $c=17.29 \AA, \alpha=48.35^{\circ}, \beta=76.6^{\circ}$ and $\gamma=62.5^{\circ}$ (chain axis along $c$ )[35-39]. The $\alpha_{I}$ form presents the most intense Bragg reflections from 100 at $2 \theta \approx 20^{\circ}$ (for an incident wavelength $\lambda=1.54 \AA$ ), corresponding approximately to the distance between hydrogen-bonded chains, and from 010 at $2 \theta \approx 24^{\circ}$, corresponding approximately to the separation between sheets formed by hydrogen-bonded chains. The distance of chain axis within the hydrogen-bonded sheets is $\approx 4.8 \AA$. A complete list of the observed Bragg reflections for the $\alpha_{I}$ form at $24{ }^{\circ} \mathrm{C}$ (after annealing at $240{ }^{\circ} \mathrm{C}$ ) is reported in Table I.

The $\alpha_{I I}$ form [1], obtained by fast cooling from the melt, has a slightly lower density $\left(\rho=1.16\right.$ g. $\left.\mathrm{cm}^{-3}\right)$ and can be distinguished from the $\alpha_{I}$ form from the distance in the reciprocal space between the 100 and 010 Bragg reflections, which, in terms of scattering angle $2 \theta$, is about $3.55^{\circ}$ for $\alpha_{I}$ and $0.72^{\circ}$ for $\alpha_{I I}$ (for $\lambda=1.54 \AA$ ). The very small 


\begin{tabular}{ccc}
\hline$h k l$ & $2 \theta\left(^{\circ}\right)$ & $Q\left(\AA^{-1}\right)$ \\
\hline 001 & 6.6 & 0.47 \\
002 & 13.5 & 0.96 \\
100 & 20.2 & 1.43 \\
010,110 & 24.0 & 1.70 \\
017 & 37.8 & 2.64 \\
117,027 & 41.0 & 2.86 \\
$\overline{1} 17$ & 46.0 & 3.19 \\
020,220 & 49.6 & 3.42 \\
\hline
\end{tabular}

TABLE I: Main Bragg reflections for PA6,6 in the $\alpha_{I}$ crystalline form at $24{ }^{\circ} \mathrm{C}$ (after Starkweather and Jones [36]), for $\lambda=1.54 \AA$. Chain axis is along $c$.

distance between the 100 and 010 Bragg reflections in the $\alpha_{I I}$ phase indicates a nearly pseudohexagonal phase (for which the reflections would exactly coincide).

In typical melt processing conditions, a crystalline phase with intermediate form is obtained, presenting a value of the angular spacing between the 100 and 010 Bragg reflections which is intermediate between the limiting values of the $\alpha_{I}$ and $\alpha_{I I}$ phases. During storage at room temperature, this intermediate phase slowly evolves toward the stable $\alpha_{I}$ phase. The deviation from the most stable $\alpha_{I}$ phase (expressed through the angular spacing between the 100 and 010 Bragg reflections) is sometimes referred to as index of crystalline perfection (ICP) $[1,40,41]$.

At length scales beyond the unit cell, semi-crystalline polyamides present a crystalline arrangement corresponding to folded-chain lamellae which organise into stacks, with part of the amorphous phase occupying the spaces between the lamellae and between the stacks [5]. The stacks themselves organise into spherulite structures dispersed in the remaining amorphous component [1]. The crystalline structure inside a spherulite has been characterized by small angle X-ray scattering (SAXS) [20, 28]. 

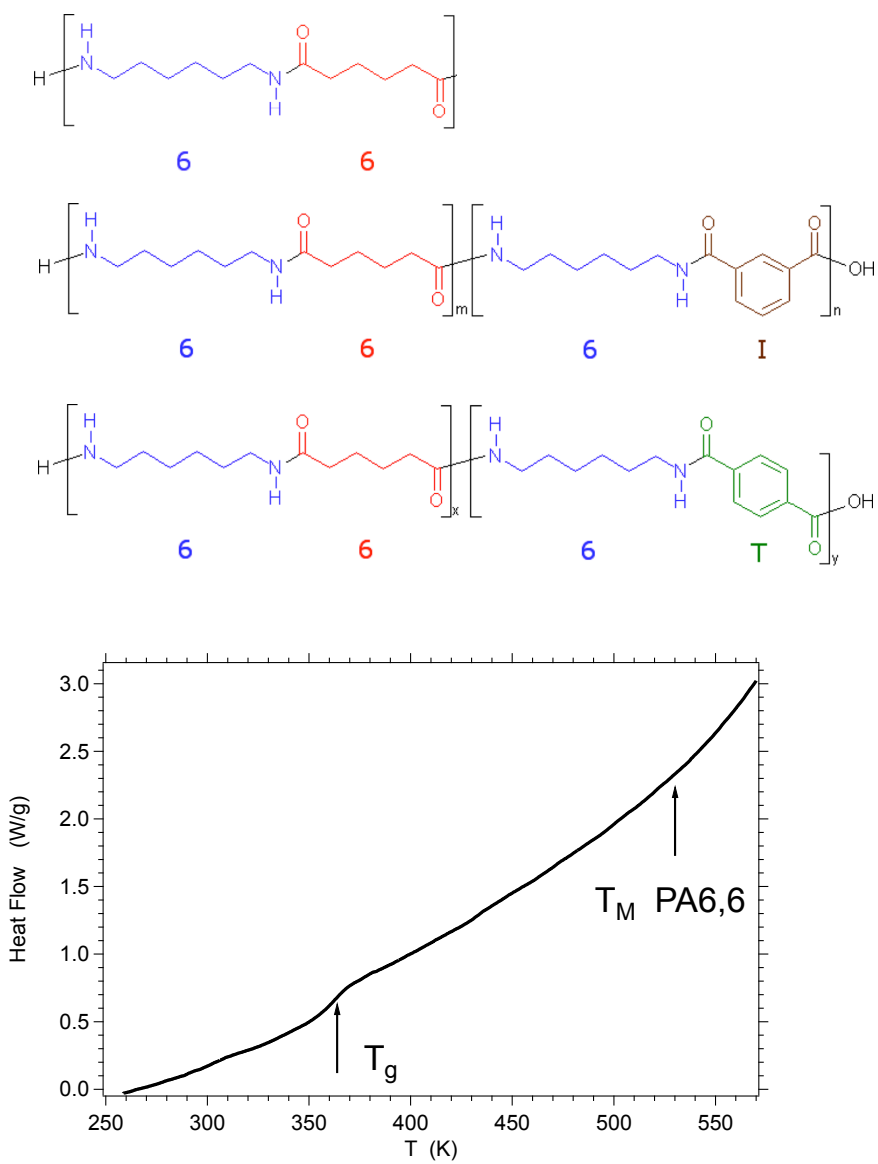

FIG. 2: a) Chemical Formulas of PA6,6 and the random copolymers PA6,6/6I and PA6,6/6T. b) Heat Flow trace measured by DSC for the PA6,6/6I copolymer, in the dry state. The glass transition temperature $T_{g}$ of $\mathrm{PA} 6,6 / 6 \mathrm{I}$ and the melting temperature $T_{M}$ of PA6,6 are indicated by arrows.

\section{MATERIALS AND METHODS}

\section{A. Samples}

Polyamide 6,6 (PA6,6) and the aromatic random copolymers PA6,6/6I and PA6,6/6T were provided by Solvay. Figure 2a) shows their chemical formulas. These copolymers are formed by condensation of hexamethylenediamine with a diacid: Adipic acid for PA6,6 and isophtalic (resp. terephtalic) acid for PA6I (resp. PA6T). PA6,6/6I contains 60\% PA6I comonomers, PA6,6/6T 35\% PA6T comonomers. The average molecular weight of the 
copolymers is about $M_{w}=10 \mathrm{~kg} / \mathrm{mol}$, with $M_{w} / M_{n}=2$. The degree of crystallinity of the semi-crystalline copolymers was estimated as $\chi_{\mathrm{c}}=\left(\Delta H_{\mathrm{m}}-\Delta H_{\mathrm{rec}}\right) / \Delta H_{\mathrm{m}}^{100 \%}$, where the melting and recristallization enthalpies, $\Delta H_{\mathrm{m}}$ and $\Delta H_{\mathrm{rec}}$ respectively, were experimentally determined by modulated Differential Scanning Calorimetry (DSC), and $\Delta H_{\mathrm{m}}^{100 \%}$ is the reference enthalpy associated with a 100\% crystalline sample. For both PA6,6 and PA6,6/6T we used for $\Delta H_{\mathrm{m}}^{100 \%}$ the value reported in Ref. [42]. We obtained $\chi_{\mathrm{c}}^{66} \approx 38 \%$ and $\chi_{\mathrm{c}}^{6 T} \approx 31 \%$. In PA6,6/6T, the 6T monomers can cocrystallize with the 6,6 monomers [43-46], but the fraction of $6 \mathrm{~T}$ monomers in the crystalline phase cannot be determined. Hence the fraction of $6 \mathrm{~T}$ monomers contained in the amorphous phase can be estimated, knowing the crystalline fraction, as being comprised between $40 \%$, in case of perfect cocrystallization of 6,6 and $6 \mathrm{~T}$ monomers, or $60 \%$ in case of crystallisation of only 6,6 monomers. The fraction of $6 \mathrm{~T}$ units in the amorphous phase is therefore comparable to that of $6 \mathrm{I}$ units in PA6,6/6I.

PA6,6 and PA6,6/6T copolymers were provided to us in the form of films of thickness $100 \mu \mathrm{m}$ and $500 \mu \mathrm{m}$ respectively. The films were obtained by extrusion using a Leistritz co-rotating twin screw extruder (diameter $D=34 \mathrm{~mm}$, ratio $L / D=35$, screw speed 255 rpm, throughput $10 \mathrm{~kg} / \mathrm{h}$ and extrusion temperature $284^{\circ} \mathrm{C}$ at the die). A planar dye was placed at the end of the extruder and the resulting product was drawn at $2 \mathrm{~m} / \mathrm{min}$ with a draw ratio of 7 through a series of rolls set at $135^{\circ} \mathrm{C}$ The pelletized materials used for extrusion were first dried in a primary vacuum oven at $110{ }^{\circ} \mathrm{C}$ for 36 hours. The water content measured by coulometric Karl Fisher titration was in the range 700 to $900 \mathrm{ppm}$. The amorphous copolymer PA6,6/6I was provided instead in form of a fine granulate, and films of thickness $d \approx 200 \mu \mathrm{m}$ were prepared by compression molding at $T=473 \mathrm{~K}$. The Heat Flow trace measured for a dry PA6,6/6I sample by DSC at $5 \mathrm{~K} \cdot \mathrm{min}^{-1}$ is shown in Fig.2b) and evidences no presence of crystallization related peaks after polymerization (the 
melting temperature of PA6,6 is $\left.T_{M}=532 \mathrm{~K}\right)$.

The glass transition temperatures $T_{g}$ (determined by DSC) of dry and fully saturated samples (with water and ethanol) are reported in Tab.II.

\begin{tabular}{lcccc}
\hline Sample & $\mathrm{m}_{w} / \mathrm{m}_{\text {tot }}$ & $\mathrm{m}_{w} / \mathrm{m}_{a m}$ & $\mathrm{~m}_{\text {mol }}$ & $\mathrm{T}_{g}(\mathrm{~K})$ \\
\hline PA6,6/6I & 0.000 & 0.000 & 0.00 & 363 \\
PA6,6/6I H ${ }_{2} \mathrm{O}$ & 0.125 & 0.125 & 0.83 & 277 \\
PA6,6/6I Eth & 0.217 & 0.217 & 0.49 & 260 \\
PA6,6/6T & 0.000 & 0.000 & 0.00 & 349 \\
PA6,6/6T H $\mathrm{H}_{2} \mathrm{O}$ & 0.073 & 0.105 & 0.72 & 273 \\
PA6,6/6T Eth & 0.095 & 0.146 & 0.37 & 260 \\
PA6,6 & 0.000 & 0.000 & 0.00 & 337 \\
PA6,6 $\mathrm{H}_{2} \mathrm{O}$ & 0.096 & 0.152 & 0.95 & 252 \\
PA6,6 Eth & 0.119 & 0.189 & 0.47 & 236 \\
\hline
\end{tabular}

TABLE II: Water mass content $\left(\mathrm{m}_{w}\right)$ relative to total $\left(\mathrm{m}_{\text {tot }}\right)$ and to the mass of the amorphous fraction $\left(\mathrm{m}_{a m}\right)$, corresponding molar content $\left(\mathrm{m}_{m o l}\right)$ relative to the number of amide groups $(\mathrm{CONH})$ in the amorphous phase and glass transition temperature $\mathrm{T}_{g}$ of the samples investigated.

\section{B. Absorption Curves}

The absorption of solvent was followed at $T=23^{\circ} \mathrm{C}$ by measuring the total mass of the sample as a function of immersion time. The weight at different immersion times was quickly measured after taking out the sample from the immersion medium and removal of excess solvent from the surface. The sample was immediately after reimmersed in the solvent. Solvent desorption during the weighting procedure can be neglected. The mass fractions of solvent (water or ethanol) in fully saturated samples are reported in Tab.II. The molar content $m_{m o l}$ refers to the number of solvent molecules per amide group in the amorphous phase. 


\section{Small Angle X Ray Scattering}

\section{Experiments}

The SAXS experiments were conducted on a Rigaku 3-pinhole PSAXS-L equipment operating at $45 \mathrm{kV}$ and $0.88 \mathrm{~mA}$. The MicroMax-002+ XRay Generator System is composed by a microfocus sealed tube source module and an integrated X-Ray generator unit which produces $\mathrm{CuK}$ transition photons of wavelength $=1.54 \AA$. The flight path and the sample chamber in this equipment are under vacuum. The scattered X-Rays are detected on a two-dimensional multiwire X-Ray Detector (Gabriel design, 2D-200X). This gas-filled proportional type detector offers a $200 \mathrm{~mm}$ diameter active area with c.a. 200 micron resolution. The azimuthally averaged scattered intensities were obtained as a function of scattering vector $Q=(4 \pi / \lambda) \sin \theta$, where $\theta$ is half the scattering angle. Reciprocal space calibration was done using silver behenate as standard. Three sample to detector distances D were employed, covering overlapping Q-ranges: $0.008-0.15 \AA^{-1}(\mathrm{D}=2 \mathrm{~m}), 0.05-0.8 \AA^{-1}(\mathrm{D}=50 \mathrm{~cm})$ and $0.1-1.7 \AA^{-1}(\mathrm{D}=24 \mathrm{~cm})$. Note that with the shortest $\mathrm{D}$ the usual WAXS range is partially accessed. All measurements were performed at room temperature and in transmission geometry. Films of the dry or dried samples were fixed perpendicular to the beam (beam section: $1.3 \mathrm{~mm}$ ) without sample holder. For the kinetic measurements, strips of the pristine films were put in capillaries of $2 \mathrm{~mm}$ thickness, which were subsequently filled with the corresponding solvent (either water or ethanol), sealed and fixed perpendicular to the beam. The first kinetic measurement was performed after about 20 minutes of the immersion (time needed to pump the air from the flight path and sample chamber). Measuring times of 10 min were employed.

Scattering length densities of the crystalline and amorphous phases, in the dry and wet 
states, are reported in Table III. They were calculated on the basis of the scattering lengths, compositions and densities of the different phases. Absorption of solvent exclusively in the amorphous phase was assumed, and the relative solvent content in the amorphous phase was calculated based on estimates of the crystalline fraction of the samples [1]. Corresponding contrast factors between crystalline and amorphous phases, in the dry and wet states, are reported in Table IV.

\begin{tabular}{lccc}
\hline Phase & $\begin{array}{c}\text { density } \\
{\left[\mathrm{g} . \mathrm{cm}^{-3}\right.}\end{array}$ & $\begin{array}{c}\text { solvent molar content } \\
(\text { refered to amid groups) }\end{array}$ & $\begin{array}{c}\sigma \\
{\left[1 \mathrm{~cm}^{-2}\right]}\end{array}$ \\
\hline Crystal $\alpha_{I}$ & 1.21 & 0.00 & 1.1239 \\
Crystal $\alpha_{I I}$ & 1.16 & 0.00 & 1.0775 \\
Dry amorphous PA6,6 & 1.09 & 0.00 & 1.0124 \\
Water-saturated amorphous PA6,6 & & 0.95 & 1.0698 \\
Ethanol-saturated amorphous PA6,6 & & 0.47 & 0.9571 \\
Dry amorphous PA6,6/6T* & 1.09 & 0.00 & 1.0042 \\
Water-saturated PA6,6/6T & & 0.72 & 1.0477 \\
Ethanol-saturated PA6,6/6T & & 0.33 & 0.9651 \\
Dry amorphous PA6,6/6I* & 1.09 & 0.00 & 0.9952 \\
Water-saturated PA6,6/6I & & 0.93 & 0.9986 \\
Ethanol-saturated PA6,6/6I & & 0.36 & 0.9582 \\
\hline
\end{tabular}

TABLE III: Scattering length densities $\sigma$ of the various phases present in the materials $\left(\mathrm{in}^{-2} \mathrm{~cm}^{-2}\right.$, calculated by taking $\beta^{2}=7.90 \times 10^{-26} \mathrm{~cm}^{2}$ for the electronic scattering cross section.*: electron density calculated by assuming that the amorphous phase density is the same as for PA6,6.

\section{Data modeling}

Following the approach of Murthy and coworkers [20, 28], we modelled the SAXS data using the following expression:

$f(Q)=a_{0}+\left(a_{1} /\left(Q^{2}+a_{2}^{2}\right)\right)+\frac{1}{Q^{2}}\left[a_{3} \exp \left(-\frac{1}{2}\left(\frac{Q-Q_{0}}{a_{4}}\right)^{2}\right)+a_{3} \exp \left(-\frac{1}{2}\left(\frac{Q-2 Q_{0}}{2 a_{4}}\right)^{2}\right)\right]^{2}$ 


\begin{tabular}{|c|c|}
\hline Contrast & $\Delta \rho^{2}\left[10^{20} \mathrm{~cm}^{-4}\right]$ \\
\hline Crystal $\alpha_{I}$-Dry amorphous PA6,6 & 1.24 \\
\hline Crystal $\alpha_{I I}$-Dry amorphous PA6,6 & 0.42 \\
\hline Crystal $\alpha_{I}$-Water-saturated amorphous PA6,6 & 0.29 \\
\hline Crystal $\alpha_{I I^{-}}$Water-saturated amorphous PA6,6 & 0.006 \\
\hline Crystal $\alpha_{I}$-Ethanol-saturated amorphous PA6,6 & 2.78 \\
\hline Crystal $\alpha_{I I}$-Ethanol-saturated amorphous PA6,6 & 1.45 \\
\hline Crystal $\alpha_{I}$-Dry amorphous PA6,6/6T* & 1.43 \\
\hline Crystal $\alpha_{I I}$-Dry amorphous PA6,6/6T* & 0.54 \\
\hline Crystal $\alpha_{I^{-}}$Water-saturated PA6,6/6T & 0.58 \\
\hline Crystal $\alpha_{I I^{-}}$Water-saturated PA6,6/6T & 0.08 \\
\hline Crystal $\alpha_{I}$-Ethanol-saturated PA6,6/6T & 2.52 \\
\hline Crystal $\alpha_{I I}$-Ethanol-saturated PA6,6/6T & 1.26 \\
\hline Crystal $\alpha_{I}$ Dry amorphous PA6,6/6I* & 1.57 \\
\hline Crystal $\alpha_{I I}$-Dry amorphous PA6,6/6I* & 0.62 \\
\hline Crystal $\alpha_{I}$ Water-saturated PA6,6/6I & 0.50 \\
\hline Crystal $\alpha_{I I}$-Water-saturated PA6,6/6I & 0.06 \\
\hline Crystal $\alpha_{I}$-Ethanol-saturated PA6,6/6I & 2.75 \\
\hline Crystal $\alpha_{I I}$-Ethanol-saturated PA6,6/6I & 1.42 \\
\hline
\end{tabular}

TABLE IV: Contrast factors $\Delta \rho^{2}$ between crystalline (both $\alpha_{I}$ and $\alpha_{I I}$ ) and amorphous phases, in the dry and wet states. ${ }^{*}$ : electron density calculated by assuming that the amorphous phase density is the same as for PA6,6.

Note that we limit our description of the experimental curves to the region of the main peak (Fig. 3), since we are primarily interested in the relative variations of the lamellar period and coherence length induced by the presence of solvent. This region is described by the last term in square brackets of equation 1 , where the peak position is denoted $Q_{0}$ and determines the period of the lamellar stacking, $L_{p}=2 \pi / Q_{0}[20,28]$. Small shifts in $L_{p}$ due to the structure factor of the lamellar stacking are neglected. The peak amplitude is described by the parameter $a_{3}$. The shape of the peak is chosen to be gaussian $[20,28]$, with a width $a_{4}$ related to the coherence length $\xi=4 \pi /\left(a_{4} \sqrt{8 \ln 2}\right)$ of the lamellar periodic stacking. Given the geometry of the experiment, $\xi$ corresponds to the coherence length along the normal to the lamellae (not the lateral extension of the lamellae). A second harmonic 
term, centred at $2 Q_{0}$ (second term in the square brackets), was included in order to account for the broadening of the peak observed on the high $Q$ side of the peak $[20,28]$. This term has to be broader than the first order peak because the disorder along the lamellar periodic stacking must be of the first kind [47]. The $1 / Q^{2}$ factor multiplying the Gaussian functions corresponds to the Lorentz correction factor [48].

\section{Wide Angle X-Ray Scattering}

Wide Angle X-Ray scattering measurements were performed on a Bruker D8 Advance diffractometer working in parallel beam geometry. With the help of a Göbel mirror, the originally divergent incident $\mathrm{X}$-ray beam from a line focus $\mathrm{X}$-ray tube $(\mathrm{Cu}$, operating at $40 \mathrm{kV}$ and $40 \mathrm{~mA}$ ) is transformed into an intense and parallel beam that is free of $\mathrm{K}_{\beta}$ radiation. The parallel beam optic required in the secondary beam path is achieved by an equatorial axial Soller slit of $0.2^{\circ}$. The linear detector LYNXEYE used presents an active area of $14.4 \mathrm{~mm} \mathrm{x}$ $16 \mathrm{~mm}$. Measurements were performed in reflection $(\theta-2 \theta$ configuration) varying $2 \theta$ from 4 to $30^{\circ}$ with a step of $0.02^{\circ}$. The total time employed was 1.8 hours/scan ( 5 seconds/point). The films were placed in an Anton Paar TTK 450 Low-Temperature chamber allowing varying the sample temperature between -193 and $450^{\circ}$ with $0.1^{\circ}$ resolution. Measurements on the dry and dried samples were performed under vacuum conditions in the chamber, and on the wet samples at ambient pressure. The data were corrected from the corresponding background signal. 


\section{E. Neutron diffraction measurements}

Neutron diffraction experiments with polarization analysis were performed at DNS (Jülich Center for Neutron Scattering (JCNS) at FRM-II, Garching, Germany). This spectrometer is capable of 3-directional polarization analysis. In this case we limited the analysis to the $z$ direction in order to separate coherent and incoherent contributions to the scattering. We used a wavelength of the incident beam $\lambda=4.8 \AA$ and the accessible $Q$ range was $0.35 \AA^{-1}<Q<2.31 \AA^{-1}$. As sample containers, we used standard flat aluminum slabs. The thickness of the films was $0.17-0.2 \mathrm{~mm}$ corresponding to calculated transmissions of 0.92-0.9. Correction for background scattering was performed by empty cell measurements. Due to the complications related to multiple scattering corrections for flat geometry and the large transmissions expected for our samples, we did not perform correction for multiple scattering. On the other hand, corrections for inelasticity effects were performed using a vanadium calibration of the incoherent scattering cross section according to the procedure described in [49].

\section{RESULTS AND DISCUSSION}

\section{A. Structure at Equilibrium}

\section{Small angle $X$-ray scattering}

Fig. 3 presents results obtained for samples in the dry state, and after long-time immersion (1 year) in water and ethanol. The intensities are expressed in arbitrary units. The peak around $0.08 \AA^{-1}$ corresponds to the lamellar stacking. It was modelled using eq.1 and the resulting fit curves are shown in Figure 3. The period $L_{p}$ and coherence length $\xi$ of the 
lamellar stacking, obtained from the fitting parameters $Q_{0}$ and $a_{4}$ in eq.1, are reported in Table V. We discuss first the results obtained for the dry samples and then, using these as a reference, the effects of solvent absorption on the observed structures.

\begin{tabular}{lcc}
\hline Sample & $L_{p}[\AA] \xi[\AA]$ \\
\hline PA6,6 dry & 69.0 & 197.6 \\
PA6,6 $\mathrm{H}_{2} \mathrm{O}$ & 73.5 & 174.5 \\
$\mathrm{PA} 6,6$ ethanol & 79.9 & 202.2 \\
\hline $\mathrm{PA} 6,6 / 6 \mathrm{~T}$ dry & 78.8 & 210.0 \\
$\mathrm{PA} 6,6 / 6 \mathrm{~T} \mathrm{H}_{2} \mathrm{O}$ & 83.5 & 222.4 \\
$\mathrm{PA} 6,6 / 6 \mathrm{~T} \mathrm{D}_{2} \mathrm{O}$ & 83.7 & 222.9 \\
$\mathrm{PA} 6,6 / 6 \mathrm{~T}$ ethanol & 89.4 & 235.3 \\
\hline $\mathrm{PA} 6,6 / 6 \mathrm{I} \mathrm{H}_{2} \mathrm{O}$ & 80.3 & 220.8 \\
$\mathrm{PA} 6,6 / 6 \mathrm{I} \mathrm{D}_{2} \mathrm{O}$ & 78.5 & 213.4 \\
PA6,6/6I ethanol & 81.6 & 213.5 \\
\hline
\end{tabular}

TABLE V: Period $L_{p}=2 \pi / Q_{0}$ and coherence length $\xi=4 \pi /\left(a_{4} \sqrt{8 \ln 2}\right)$ along the normal to the lamellae, of the periodic lamellar stacking, obtained from the fitting parameters, $Q_{0}$ and $a_{4}$, determined by modelling experimental data of figure 3 using equation 1 ,

a. Dry samples For dry PA6,6 we obtain a period of the lamellar stacking $L_{p} \approx 69 \AA$, and a coherence length $\xi \approx 198 \AA$, as reported in Table $\mathrm{V}$. The $Q$ range of the SAXS data shown in Figure 3 extends to large enough $Q$ values, such that the first peaks corresponding to Bragg reflections of the crystalline unit cells are also observed. These are the peaks observed at $Q \approx 0.47$ and $Q \approx 0.95 \AA^{-1}$, corresponding to the 001 and 002 Bragg reflections of the $\alpha_{I}$ crystalline form of PA6,6 respectively (see Table I) and the more intense peak at $Q \approx 1.5 \AA^{-1}$, which contains the contributions from the 100, 010 and 110 Bragg reflections and also the contribution of the structural arrangements of the chains on the atomistic level in the amorphous phase (amorphous ring).

The PA6,6/6T copolymer in the dry state (Figure 3(b)) shows a spectrum very similar to that of PA6,6. The main difference with respect to PA6,6 is the position of the main (lamellar) peak, which is shifted to a slightly lower $Q$ value, corresponding to a larger lamellar 


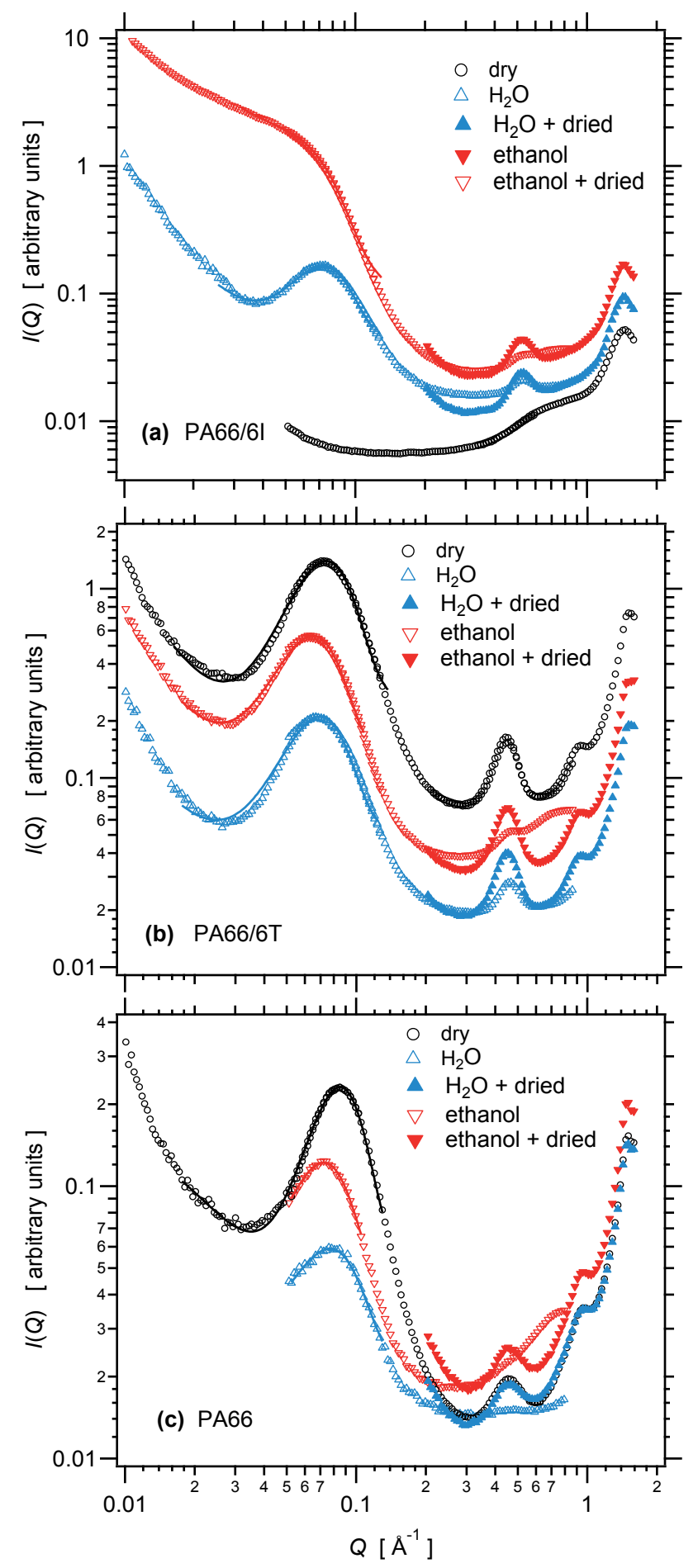

FIG. 3: Small angle X-ray scattering spectra of as prepared, dry samples (black circles) and of samples after one year time immersion in $\mathrm{H}_{2} \mathrm{O}$ (empty blue triangles) and ethanol (empty red down triangles), for (a) PA6,6/6I (b) PA6,6/6T (c) PA6,6. Solid symbols correspond to spectra of samples dried back from the saturated state. Note that intensities have not been normalized with respect to each other and spectra have been shifted vertically (in log scale) in order to be better separated. Curves are fits of the peak obtained using equation 1. 
period $L_{p} \approx 78.8 \AA$.

On the other hand, the dry PA6,6/6I copolymer shows a completely different spectrum. Both the peak corresponding to the lamellar order and the 001 and 002 Bragg reflections are absent (Figure 3(a)). This indicates that the polymer is amorphous. The very small shoulder at $Q \approx 0.6 \AA^{-1}$ and the peak at about $1.5 \AA^{-1}$ are contributions from the amorphous phase, as also confirmed by atomistic simulations [50].

b. Long-term effects of solvent immersion Addition of water to the PA6,6 and PA6,6/6T polymers leads to a shift of the main peak to lower $Q$ values (Figures 3(b) and (c), Table V), or equivalently to an increase of the lamellar periods $L_{p}$ of 5-6\% (from 69 to $73.5 \AA$ in PA6, 6 and from 78.8 to $83.6 \AA$ in PA6,6/6T), which can be attributed to the fact that water occupies the interlamellar space and induces a swelling, as already observed for PA6,6 and PA6 [27, 28]. Interestingly, the coherence length decreases with water absorption in PA6,6, but slightly increases in PA6,6/6T (Table V).

Assuming rigid crystalline lamellae and thus $1 \mathrm{D}$ swelling of the lamellar stacking, the increase of the lamellar period would correspond to an overall volume variation of lamellar stackings of about 5-6\%. The material contains both amorphous domains and lamellar stacks, composed of crystalline lamellae and an amorphous phase constrained in between them [28]. It is thus interesting to compare the volume variation of lamellar stackings (assuming 1D swelling) to the overall volume variation of the swollen samples. For an overall water mass uptake of the order of $15 \%$ (approximately one water molecular per amide group), the density of the amorphous phase changes from 1.09 g.cm ${ }^{-3}$ to approximately 1.15 g.cm ${ }^{-3}$ and the overall volume change of the sample is approximately $9 \%$ (assuming the solvent is absorbed exclusively in the amorphous phase). Thus, this comparison gives quite consistent 
figures. It suggests that water would swell preferentially the amorphous domains.

Note in addition that the Bragg reflections 001 and 002 become smoother in the wet state, which indicates that solvents have an impact on the crystalline structure itself inducing a modulation of the electron density within the crystal. This effect will be discussed in more detail in the next section.

The spectrum of water saturated PA6,6/6I changes drastically compared to that of the dry polymer. The originally amorphous polymer develops a crystalline order similar to that observed for PA6,6 and PA6,6/6T, as proven by the appearance of a peak from the lamellar structure (Figure 3(a)) as well as of a 001 Bragg reflection around $0.5 \AA^{-1}$. The lamellar period and coherence length of water saturated PA6,6/6I correspond approximately to those of wet PA6,6/6T (Table V), but the 001 Bragg reflection is slightly shifted towards higher $Q$ values (by about $10 \%$ ) compared to the $\alpha_{I}$ crystalline form in PA6,6 and PA6,6/6T. This corresponds to a slightly smaller $c$ value (less stretched chains) in the crystalline mesh. Considering a similar crystal density, distances between chains in the crystal should then be slightly larger. This may be associated to a more disordered crystalline phase in PA6,6/6I than in PA6,6 and PA6,6/6T. Note also that in PA6,6/6I the 002 Bragg reflection is barely visible, which supports the last statement.

The effect of addition of ethanol to PA6,6 and PA6,6/6T is similar to that of water: An increase in the lamellar period, which is more pronounced than for water, as indicated by the shift of the main peak to even lower $Q$ values (Figures 3b,c and 3c) and the corresponding extracted values of $L_{p} \approx 79.9 \AA$ in PA6,6 and $89.4 \AA$ in PA6,6/6T. This is in qualitatitive agreement with the larger increase of volume in ethanol saturated samples. Additionally, the coherence length increases further with respect to the case of water absorption. Note also that the 001 and 002 Bragg reflections values are reduced in intensity, similar to what 
was found in presence of water.

In PA6,6/6I, the addition of ethanol induces the appearance of a peak corresponding to the lamellar stacking, which in this case is partially masked by a strong forward scattering of the amorphous phase. The appearance in addition of a 001 Bragg reflection (Fig. 3(a)) indicates without ambiguity that immersion in ethanol also induces some crystallinity in the originally amorphous PA6,6/6I. Note again that the 002 Bragg reflection is barely visible. Differently from PA6,6 and PA6,6/6T, the lamellar spacing and coherence length in ethanol are essentially comparable to the ones obtained for water immersion, which might be related to the development of crystallisation in the presence of solvent in the case of PA6,6/6I, as will be discussed later in the manuscript.

All wet samples were subsequently dried under vacuum at $T=90^{\circ} \mathrm{C}$. PA6,6 and PA6,6/6T samples essentially recover the same features observed in their initial (as prepared) dry state. Remarkably, the semi-crystalline order which has been induced in the PA6,6/6I sample upon solvent immersion is preserved under subsequent solvent removal, as shown in Figure 3(a) through the presence of the lamellar peak and the 001 and 002 Bragg reflections in the solvated, then dried sample.

\section{Wide angle $X$-ray scattering}

In order to investigate in detail solvent-induced changes in the structure at a local scale where the atomistic details of the chain composition determine the scattering, we performed X-ray wide angle scattering measurements. Figure 4 shows the results obtained for the three polymers in the dry condition, and after long-time immersion in water and ethanol (1 year). Interestingly, whilst the SAXS study did not reveal significant changes of the 
lamellar structure of already semi-crystalline materials, with solvent absorption inducing a swelling of the interlamellar space, significant modifications of the crystalline phase are observed at the local scale for the PA6,6 and PA6,6/6T copolymers. PA6,6 presents in the dry state a crystalline structure intermediate between the $\alpha_{I}$ and the $\alpha_{I I}$ phases. The Q-spacing between the 100 and $010+110$ Bragg reflections changes in a reproducible way from $\Delta Q=0.135 \pm 0.005$ to $\Delta Q=0.20 \pm 0.002 \AA^{-1}$ (see Figure 4c) in the presence of solvent (water and ethanol have similar effects). Correspondingly, the two Bragg peaks become better resolved. This indicates that the index of crystalline perfection increases in the wet samples, or equivalently that the crystallite structure evolves towards a structure closer to the more stable $\alpha_{I}$ phase under the influence of solvents. Note that the amorphous contribution of the solvent could have some influence on the observed changes. These changes suggest that a small fraction of water penetrates into the crystallites, breaking H-bonds and facilitating the rearrangement of the crystal structure. Alternatively, or in addition, they could be attributed to a plasticization effect induced by the solvent and a consequently enhanced mobility: This possibility will be discussed in section IV D in the context of water induced crystallisation of PA6,6/6I. Modifications of the local crystalline structure in the presence of solvent are even more evident for PA6,6/6T (Figure 4b): In the dry state, the copolymer shows an almost coinciding single peak for the 100 and 010,110 reflections, at a $Q$ value intermediate between the peaks of the $\alpha_{I}$ phase. This indicates a crystalline phase compatible with the $\alpha_{I I}$ crystalline state. After absorption of water or ethanol the two reflections separate in $Q$ values, in the direction of the peaks expected for the $\alpha_{I}$ phase. This indicates formation of an intermediate phase, similar to PA6,6, which is again possibly due to the solvent induced rearrangement of the H-bond structure in the crystalline phase. The appearence of crystallinity in PA6,6/6I after absorption of solvent is visible also in 
wide angle scattering, despite the superposition of the Bragg reflections with the amorphous scattering contribution. The 001 Bragg reflection, already observed in the SAXS spectra, is also visible here. Moreover, the 002 reflection, if at all there, has a very small intensity. In the WAXS data we can now observe the details of the two peaks corresponding to the 100 and 010,110 reflections. The positions of the peaks, approximately at $1.45 \AA^{-1}$ and $1.70 \AA^{-1}$, roughly correspond to the values expected for the $\alpha_{I}$ phase. The crystallisation in the most stable form could be the result of the slow crystallisation process induced by the absorption of solvent. Comparable crystalline structures are obtained after absorption of $\mathrm{H}_{2} \mathrm{O}$ or Ethanol.

Figure 5 shows the comparison between the spectra measured by WAXS for all samples in the wet states (water or ethanol) and those measured after drying them under vacuum at $90{ }^{\circ} \mathrm{C}$. The primary observation is that the crystalline structure induced in PA6,6/6I and changes in the one of PA6,6/6T remain after drying. Small change in the overall intensity of the spectra may be attributed to the amorphous contribution of the solvent to the total scattering cross section in the wet samples. In addition, small changes are observed in the relative intensities and position of the Bragg peaks in all cases, which corresponds to subtle changes in the crystalline structure.

\section{B. Structural variation kinetics during immersion in polar solvents (SAXS)}

To investigate the kinetics of structural changes from the initial dry state to the solvent saturated state, we measured SAXS spectra of the three samples as a function of the delay time $t_{w}$ after immersion in the solvent. 


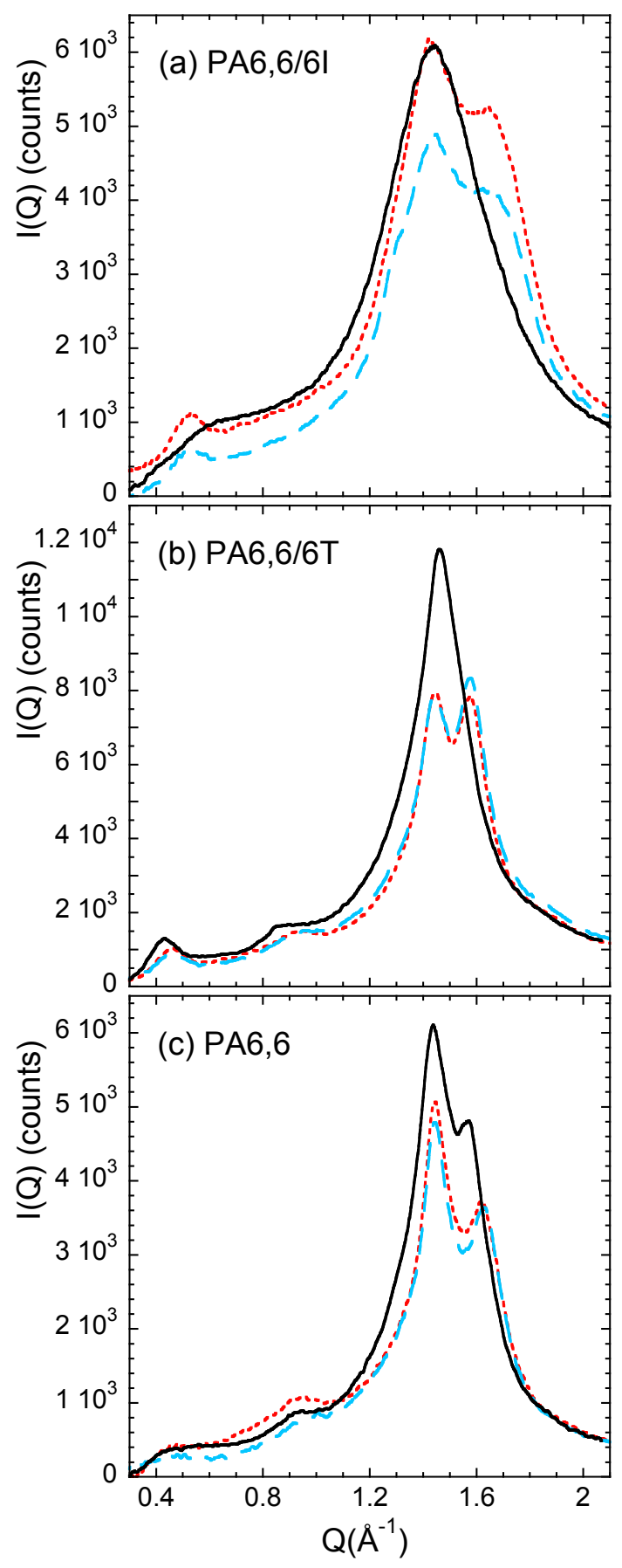

FIG. 4: WAXS results on PA6,6/6I (a), PA6,6/6T (b) and PA6,6 (c) samples at room temperature, never immersed (black solid line) and after being immersed in $\mathrm{H}_{2} \mathrm{O}$ (blue dashed line) and in ethanol (red dotted line). 


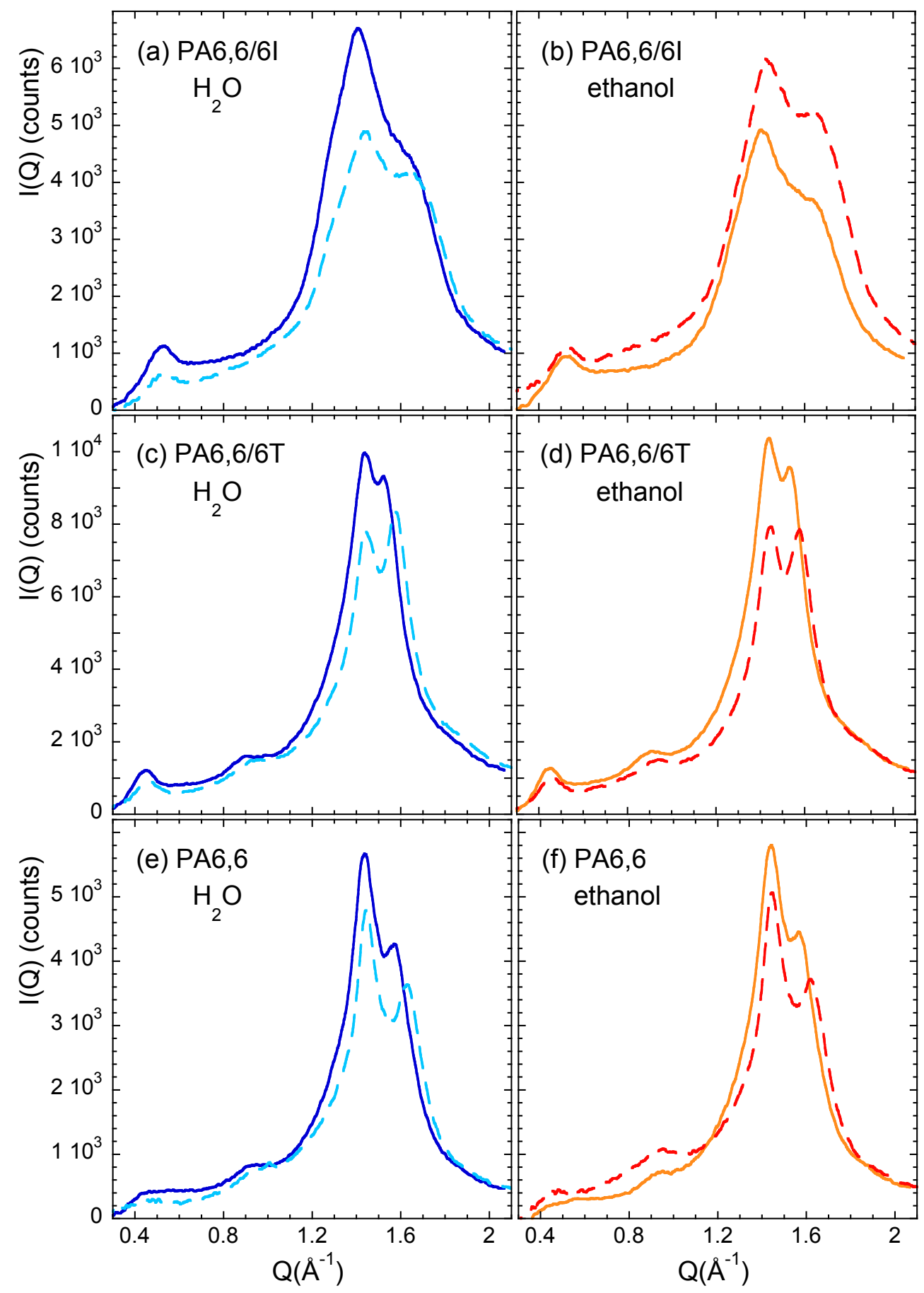

FIG. 5: WAXS results on PA6,6/6I (a,b), PA6,6/6T (c,d) and PA6,6 (e,f) samples at room temperature, wet (dashed lines) and after being dried (solid lines) in $\mathrm{H}_{2} \mathrm{O}$ (left panels a, c and e) and in ethanol (right panels $b, d$ and $f$ ). 


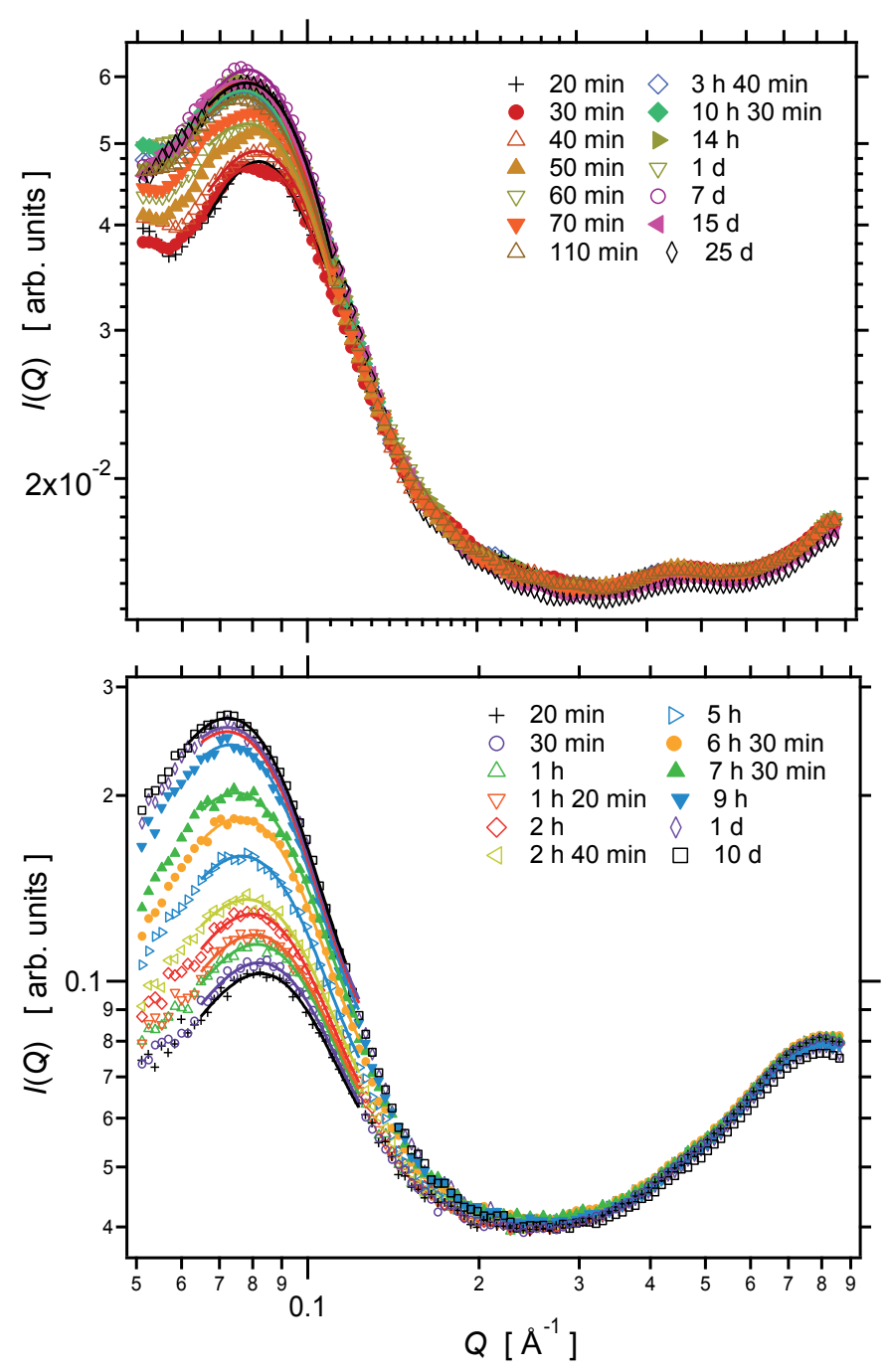

FIG. 6: SAXS spectra of PA6,6 immersed in solvent at time 0, as a function of immersion time $t_{w}$ (indicated in minutes). Top: water; bottom: ethanol. Continuous curves are fits of the lamellar peak with equation 1.'min' denotes minutes, 'h' hours and 'd' days.

\section{1. $P A 6,6$}

The SAXS spectra obtained for PA6,6 at different delay times $t_{w}$, and fits of the main peak of the spectra to the function of equation 1, are shown in Figure 6. In the presence of water (top graph in Figure 6, and left hand side column in Figure 7) the main peak corresponding to the lamellar stacking slightly shifts to lower $\mathrm{Q}$ values. The results of the 


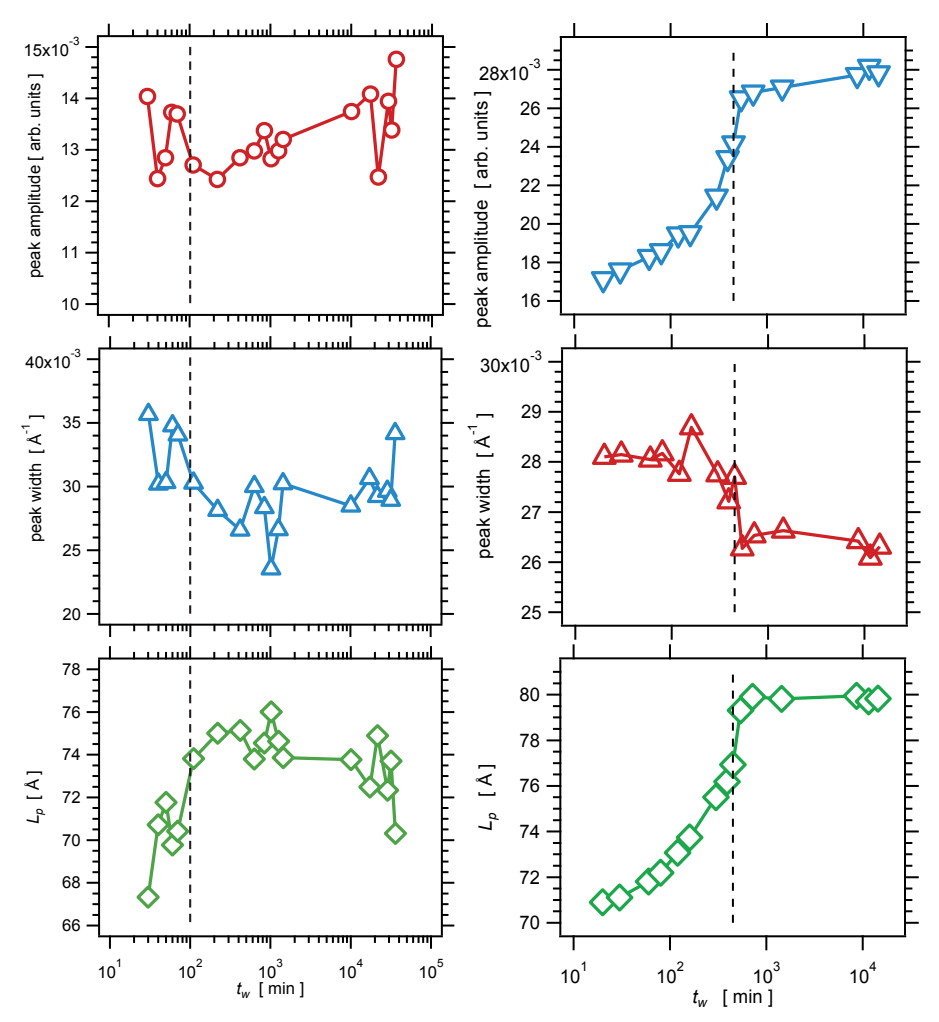

FIG. 7: Fitting parameters of the lamellar peak for PA6,6 (curves shown in Fig. 6) as a function of immersion time in solvent $t_{w}$ expressed in minutes: left column: in water (top graph in Fig. 6); right column: ethanol (bottom graph in Fig. 6). From top to bottom: peak amplitude, parameter $a_{3}$ in Eq. 1; peak width, parameter $a_{4}$ in Eq. 1 ; lamellar period $L_{p}=2 \pi / Q_{0}$. Curves are exponential fits. Dashed lines indicate the absorption time $\tau_{\text {abs }}$ from table VI.

fits of the peak position are somewhat noisy, but indicate a decrease of $Q_{0}$, i.e. an increase of the lamellar spacing over a time scale $t_{w} \approx 100$ min (Figure 7, bottom). Even though equally affected by noise, over a comparable time scale also the peak width and the amplitude show the most significant changes, with the first one decreasing and the second one increasing. The relative changes in the peak amplitude could be explained by changes in the contrast in electron densities between the amorphous and crystalline phase as water penetrates the amorphous phase, see Table IV. Note that the timescale $t_{w}$ over which some changes in the parameters of the lamellar stacking are observed is comparable to the water saturation timescale obtained from absorption kinetics (Figure 12). 
In ethanol (bottom graph in Figure 6 and right hand side column in Figure 7) the trends discussed for water are much more pronounced and less affected by noise. The evolution as a function of the immersion time $t_{w}$ exhibits two distinct regimes. In the first regime (up to $t_{w} \approx 600$ minutes), the peak amplitude increases continuously. This increase may be explained primarily by the increase of contrast as ethanol penetrates into the amorphous phase (see Table IV). This timescale can be again compared to the ethanol absorption timescale obtained from absorption kinetics measurements (Figure 12). Simultaneously, there is an increase of the lamellar period $L_{p}$, corresponding to swelling of the lamellar stacks, together with a small relative decrease of the peak width. In the second regime, parameters stay constant. This means that, apart from the effect of swelling the amorphous phase, immersion of PA6,6 in ethanol or water does not seem to induce changes in the degree of crystallinity. Note however that a small but significant effect of ethanol on the local crystalline structure was observed, as shown in Figure 4(c).

\section{2. $P A 6,6 / 6 T$}

In PA6,6/6T (Figure 8) the kinetic measurements show a progressive shift of the main peak to lower $Q$ values, i.e. the progressive increase of the lamellar period until the equilibrium value is reached, both in water (top graph in Figure 8) and in ethanol (bottom graph). Fit curves of the lamellar peak maximum obtained using equation 1 are shown in the same figure. One can observe that in this case structural changes are observed over considerably longer time scales than in PA6,6. This can be mainly attributed to the different thickness of the samples, which results in longer absorption kinetics for PA6,6/6T, while the diffusion coefficients are comparable for the two samples (Fig. 12 and Tab. VI).

The evolution of the fitting parameters obtained for PA6,6/6T as a function of the im- 


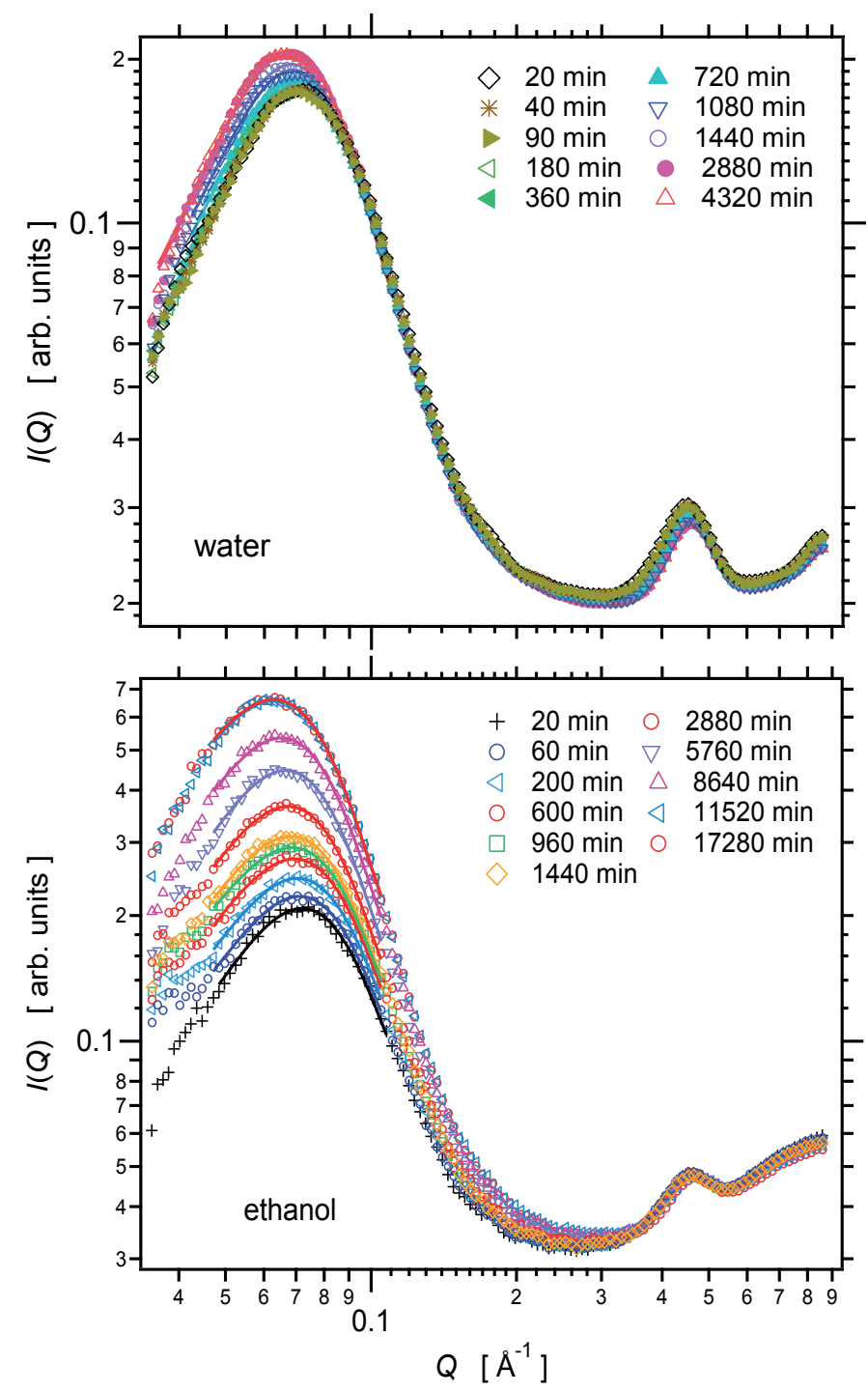

FIG. 8: SAXS spectra of PA66,6T immersed in solvent at time 0, as a function of immersion time $t_{w}$ (indicated in minutes). Top: water; bottom: ethanol. Continuous curves are fits of the lamellar peak with equation 1.

mersion time $t_{w}$ is shown in Figure 9. The increase of the lamellar period $L_{p}$ in PA6,6/6T as a function of solvent immersion, for both water and ethanol (bottom graph), can be reproduced by a single exponential function with characteristic times $\tau_{6 T}$ of approximately 700 to 900 minutes in water and 6700 to 7800 minutes in ethanol. On the other hand the peak width slightly decreases as the immersion time increases (mid graphs). This indicates an increase of the correlation length of the lamellar stacking in the presence of water or 

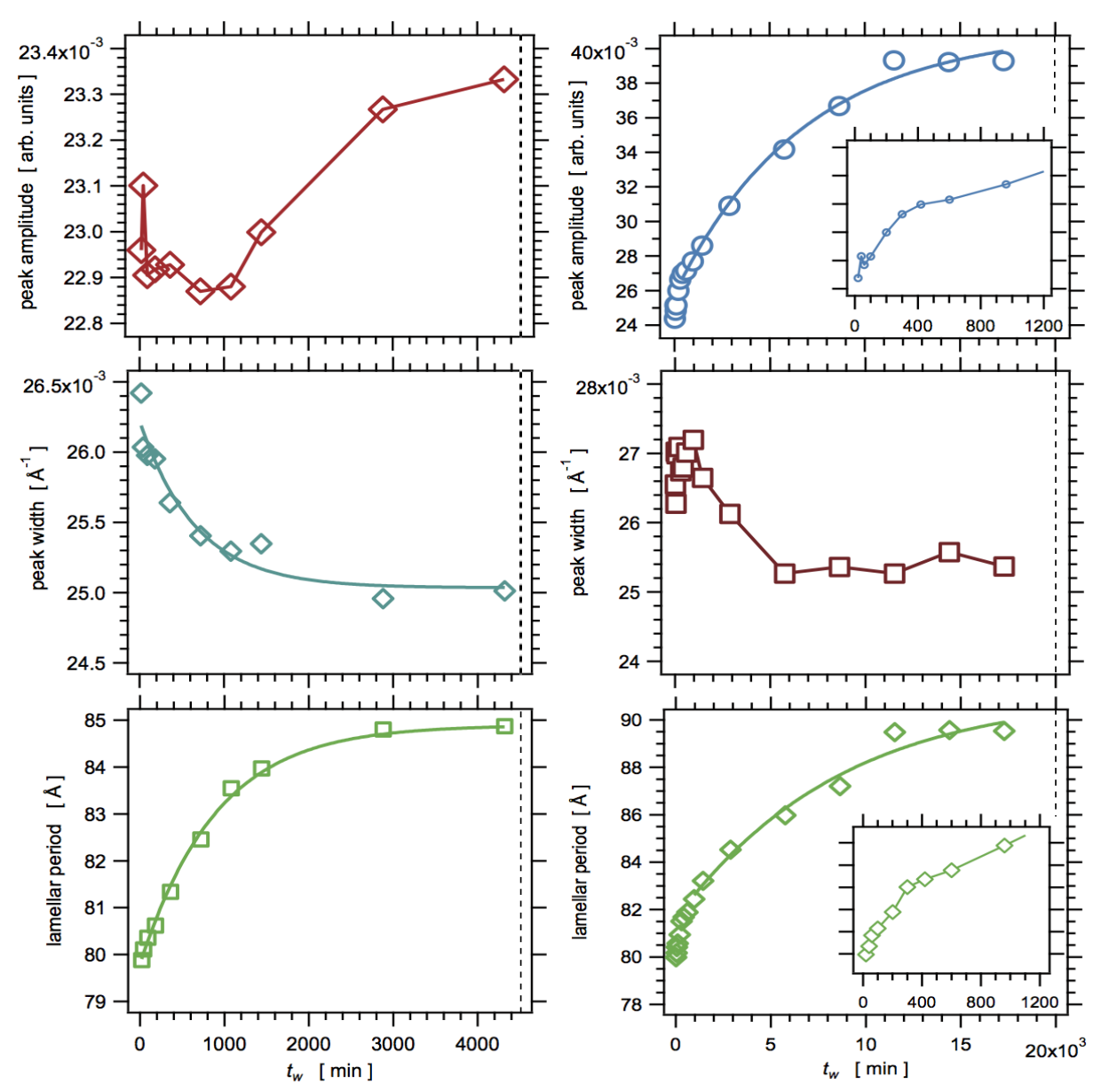

FIG. 9: Fitting parameters of the lamellar peak for PA66/6T (curves shown in Fig. 8) as a function of immersion time in solvent $t_{w}$ expressed in minutes: left column: in water (top graph in Fig. 8); right column: ethanol (bottom graph in Fig. 8). From top to bottom: peak amplitude, parameter $a_{3}$ in Eq. 1; peak width, parameter $a_{4}$ in Eq. 1; lamellar period $L_{p}=2 \pi / Q_{0}$. Curves are exponential fits. Dashed lines indicate the absorption time $\tau_{\text {abs }}$ from table VI. Insets: Log-lin representation of the data in the main plots, evidencing the two regimes in the $t_{w}$-dependence of the data.

ethanol. The typical evolution time is the same as that of the lamellar period. The peak amplitude (top graphs) also increases with increasing immersion time and in the case of ethanol immersion can be again modelled with an exponential dependence with a characteristic time comparable to that of the lamellar period. In the case of water, the variation 
of the peak amplitude at short waiting times seems to be less significant and a clear exponential dependence cannot be determined in this case. Variations of the amplitude of the peak in ethanol are qualitatively coherent with the increase of contrast with progressive absorption of ethanol, see Tab. IV. This is not true in water since a decrease of contrast with increasing water content would be expected according to the estimates in Tab. IV. This might be attributed to modulations due to the form factor of the lamellar stack and correlations between crystallites. These effects are apparently more important for the case of water absorption compared to ethanol absorption. The variations of the peak amplitude during immersion in water are still relatively small, and therefore do not seem to correspond to a significant increase of the crystallinity. On the other hand, a significant effect of ethanol on the local crystalline structure was observed, as shown in Figure 4(b)

\section{3. $P A 6,6 / 6 I$}

Figure 10 (top row) shows X-ray spectra for PA6,6/6I immersed in water, at different immersion times $t_{w}$. The initial spectrum of the amorphous polymer slowly develops a peak positioned at $Q$ values which increase with time (Figure 10, top left), until finally a crystalline phase which resembles that of semi-crystalline PA6,6 and PA6,6/6T becomes stable. Changes in the position of the main peak with immersion time indicate that the crystalline lamellar structure slowly develops.

Note that the time needed to reach a final equilibrium crystalline phase (of the order 10 days) is much longer than the time needed to saturate the sample with water (see absorption curves later, in Fig. 12). Also the peak located at the position of the 001 Bragg reflection (Figure 10, top right) develops with immersion time, simultaneously to the development of the lamellar peak. 

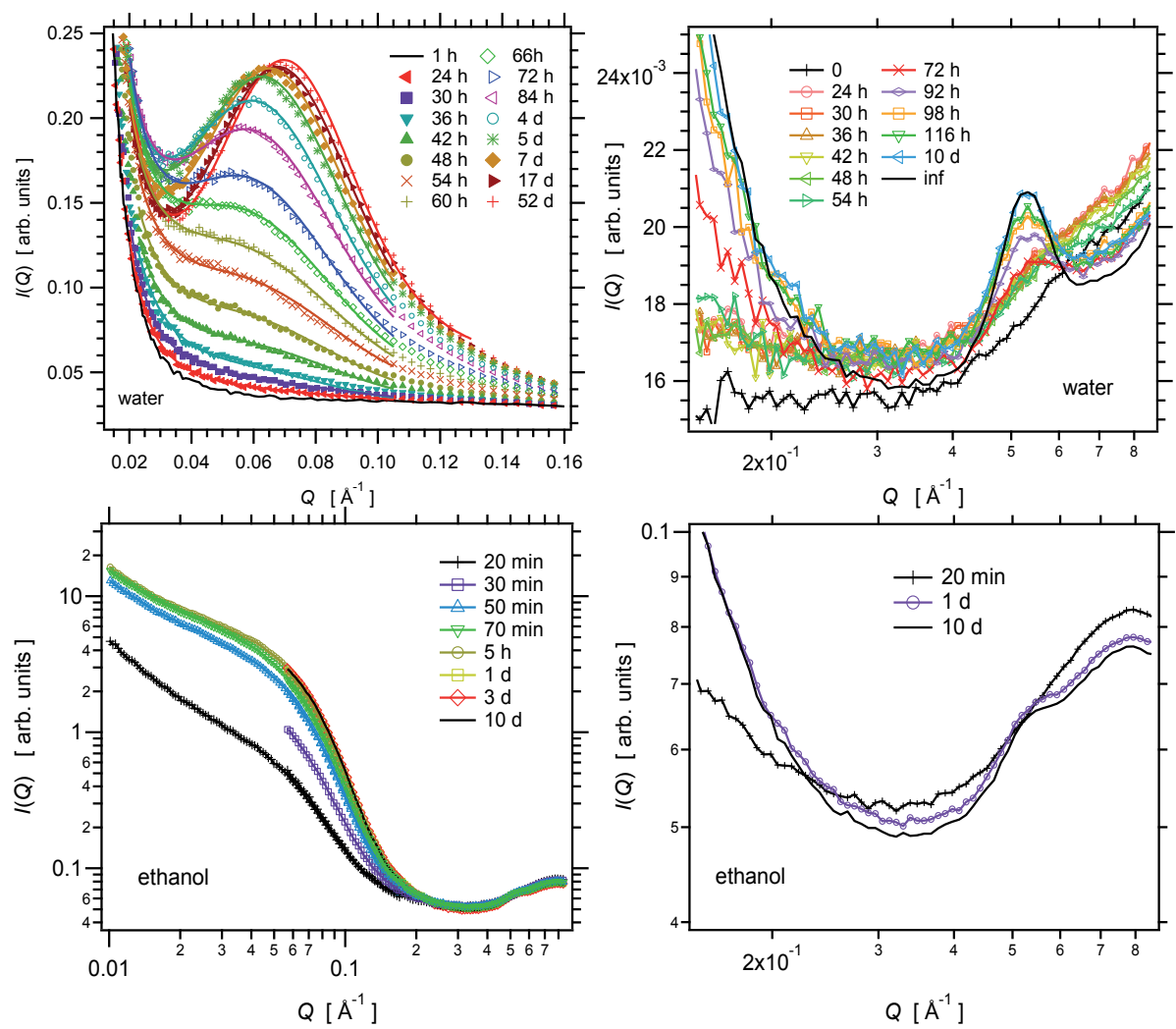

FIG. 10: SAXS spectra of PA6,6/6I immersed in solvent at time 0, as a function of immersion time $t_{w}$. Top row: water; bottom row: ethanol. Graphs in each column correspond to different $Q$ ranges to show the growth of the lamellar peak (left column) and of the 001 Bragg reflexion (right column).In the legends, 'min' denotes minutes, 'h' hours, 'd' days and 'inf' denotes one year immersion in water. In the top left graph, continuous curves are fits with equation 1.

Fitting of the lamellar peaks at various immersion times using equation 1 allows to describe the evolution of the semi-crystalline lamellar structure quantitatively. Fitting curves are shown in Figure 10 (top left graph).

The obtained variation of the fitting parameters as a function of immersion time $t_{w}$ in water for PA6,6/6I (initially amorphous) are shown in Figure 11. The amplitude of the peak 
(Figure 11, top graph) (corresponding to the fitting parameter $a_{3}$ in equation 1), is related to the relative increase of the crystallinity amount as a function of immersion time. After an initial time $t_{0} \approx 35$ hours over which no change is observed, the amplitude increases monotonically with increasing immersion time. Note that the observed increase of the peak intensity is considerably larger than contrast variations induced by the presence of water (Table IV). The peak width (Figure 11, mid graph) corresponds to the fitting parameter $a_{4}$ in equation 1 . After an initial time regime $t_{0}$, corresponding in this case to about $60-70$ hours, over which the peak region cannot be accurately fitted using equation 1 , the curve shows a monotonic decrease, associated with an increase of the coherence length of the lamellar stacking. Simultaneously, for times longer than $t_{0}$, the lamellar period $L=2 \pi / Q_{0}$ (Figure 11, bottom), where $Q_{0}$ is the fitted peak position in equation 1 , decreases as the immersion time $t_{w}$ increases.

The time dependence $f\left(t_{w}\right)$ of the peak amplitude, the width and the position (Figure 11 a,b,c) for times $t_{w}>t_{0}$ can be semi-quantitatively modelled using a double exponential function:

$$
f\left(t_{w}-t_{0}\right)=A_{1} e^{-\left(t_{w}-t_{0}\right) / \tau_{1}}+A_{2} e^{-\left(t_{w}-t_{0}\right) / \tau_{2}}
$$

where $A_{1}$ and $A_{2}$ are the relative intensities of the two components, and $\tau_{1}$ and $\tau_{2}$ the characteristic times of the exponential decays. The obtained typical evolution times are $\tau_{1} \approx 30$ hours and $\tau_{2} \approx 370$ hours, respectively. Note that full absorption takes place within the initial time regime $t_{0}\left(\tau_{\text {abs }} \approx 8\right.$ hours, see Table VI). This indicates that these two times both relate to the crystallisation process, which apparently takes place over two different timescales: Initially crystallinity and index of crystalline perfection proceed faster, but the final steady-state is reached with a slower process. 


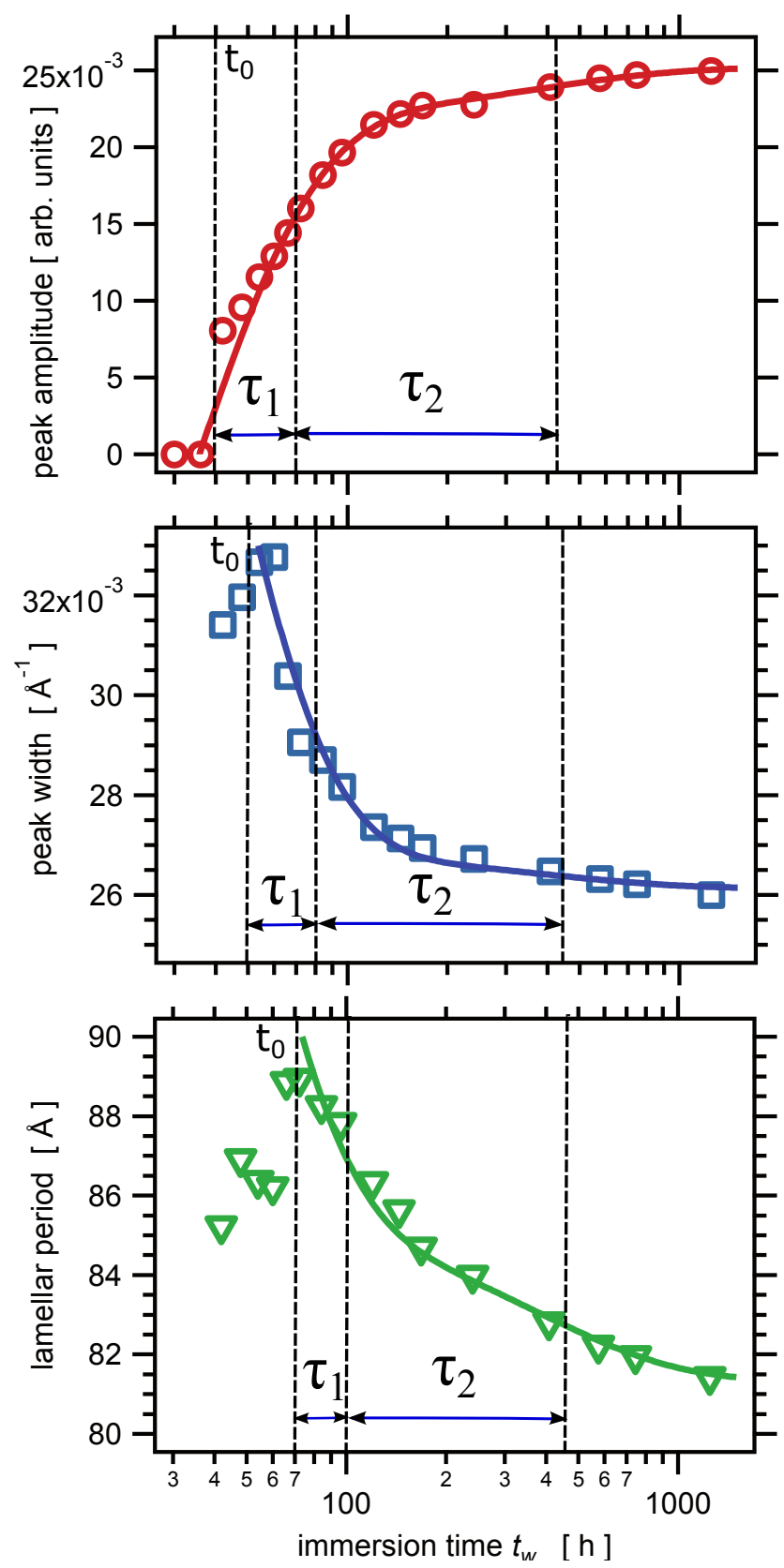

FIG. 11: Fitting parameters as a function of immersion time in water $t_{w}$ (in hours), obtained from fitting the data of Fig. 10. From top to bottom: peak amplitude, parameter $a_{3}$ in Eq. 1; peak width, parameter $a_{4}$ in Eq. 1; lamellar period $L=2 \pi / Q_{0}$, where $Q_{0}$ is the peak position in equation 1. Obtained from fitting the data shown in Fig. 10. Curves are double exponential fits. The dashed lines, from left to right, indicate the times $t_{0}, t_{0}+\tau_{1}$ and $t_{0}+\tau_{2}$. Double arrows indicate the times $\tau_{1}$ and $\tau_{2}$. 
The time evolution of the intensity of the 001 Bragg reflection (top right graph in Figure 10) evidences the formation of a clear peak after 72 hours, approximately corresponding to the time $t_{0}$ obtained for the time evolution of the main peak. Moreover the intensity of the peak increases, and its position shifts to slightly lower $Q$ values, over a time interval of 7 days after formation of the peak (after 72 hours), i.e. $\tau_{001}=168$ hours. Note that this time is intermediate between $\tau_{1}$ and $\tau_{2}$.

In ethanol (Figure 10, bottom row), the effects are qualitatively similar to those observed for water: A clear increase of the peak amplitude as a function of the immersion time is observed, corresponding to a progressive increase of the crystalline fraction, and a shift of the peak position to larger values. Again the increase in amplitude is considerably larger than variations in contrast due to solvent absorption (Table IV). In the whole investigated time range though the amplitude of the lamellar peak remains small compared to the rather strong forward scattering. Due to the relatively large uncertainties that this induces in the modelling of the data, we do not report the values of the fit parameters of the lamellar peak obtained using equation 1 in this case. It is nevertheless clear from Figure 10 (bottom left) that the timescale of development of the crystalline structure is much faster than in water, since the curves do not change significantly after $\tau_{1}^{\text {eth }}=70 \mathrm{~min}$. This faster crystallisation process might be the result of the stronger plasticisation effect of Ethanol in combination with the rearrangement of the hydrogen bond structure. Note also that the faster timescale of crystallisation during immersion in ethanol is reflected in the absorption kinetics (figure 12), as will be discussed in more detail in section IV C. The 001 Bragg reflection also develops in the presence of ethanol, though its intensity remains very small (see bottom right graph in Figure 10). In any case, together with the small amplitude of the lamellar peak, 
this qualitatively indicates that the overall crystalline fraction remains small, compared to the already semi-crystalline materials.

\section{Absorption and crystallisation}

For each sample, it is interesting to compare directly the SAXS results to absorption curves giving the time evolution of the absorbed solvent amount. These curves are shown in Figure 12. Note that the different thickness of the samples contributes to the largely different values of the absorption times $\tau_{\text {abs }}$, i.e. the times to reach solvent saturation, obtained for the different polymers.

For PA6,6, typical absorption times are of order $100 \mathrm{~min}$ for water and $520 \mathrm{~min}$ for ethanol. For water, the approximately linear increase of solvent intake with the square root of absorption time indicates Fickian diffusion, for which an effective diffusion coefficient $D_{\text {wat }}^{66}=l^{2} / \tau_{\text {abs }} \approx 1.67 \times 10^{-10} \mathrm{~m}^{2} / \mathrm{s}$ can be defined, with $l$ the sample thickness. For ethanol the absorption curve indicates significant deviations from Fickian diffusion and no diffusion coefficient was estimated. The values of the absorption times correspond well to those over which significant variations of the lamellar structure are observed, as indicated by the dashed lines in Figure 7. For PA6,6/6T the absorption times are about 4500 min (3.1 days) for water, and 20000 min (13.8 days) for ethanol. For water we can again extract an effective diffusion coefficient $D_{\text {wat }}^{6 \mathrm{~T}} \approx 0.92 \times 10^{-10} \mathrm{~m}^{2} / \mathrm{s}$, which is smaller but comparable to that obtained for PA6,6. The absorption times once more correspond to the ones over which significant changes in the scattering curves are obtained, as shown in Figure 9. The correspondence between absorption times and the kinetics of structural change measured by SAXS supports the interpretation that in the already semi-crystalline materials the water penetrates preferentially the amorphous regions, swelling them and inducing an increase of 

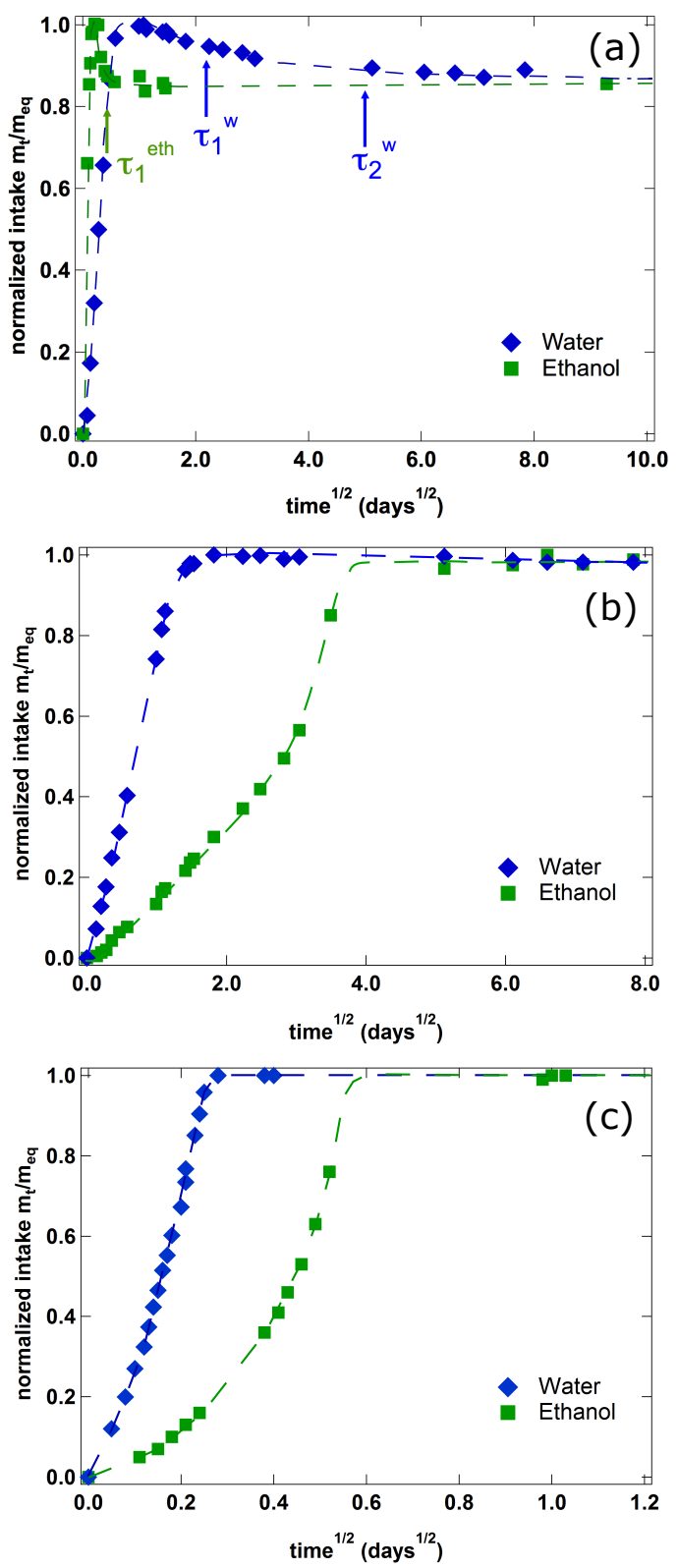

FIG. 12: Absorption curves of water and ethanol in (a) PA6,6/6I, (b) PA6,6/6T, (c) PA6,6, at $T=23^{\circ} \mathrm{C}$. Lines are guides for the eye. In (a) arrows and corresponding annotations indicate the characteristic times over which variations of the SAXS spectra in the kinetics measurements are observed, according to results of section IV B 3. Colousrs according to the legend.

the lamellar spacing. The increase of the lamellar spacing is seen as a shift of the position of the main peak of the SAXS spectra with increasing immersion time, until solvent saturation occurs and the peak does not vary any longer significantly.

The case is different for PA6,6/6I: In this case the absorption of water or ethanol induces 


\begin{tabular}{lccc} 
& & \\
\hline Sample & $\tau_{\text {abs }}^{\text {water }}[\mathrm{min}]$ & $\tau_{\text {abs }}^{\text {ethanol }}[\mathrm{min}]$ & $D_{\text {wat }}\left[10^{-0} \mathrm{~m}^{2} / \mathrm{s}\right]$ \\
\hline PA6,6 & 100 & 520 & 1.67 \\
PA6,6/6T & 4500 & 20000 & 0.92 \\
PA6,6/6I & 500 & 60 & 1.33 \\
\hline & & &
\end{tabular}

TABLE VI: Absorption times $\tau_{\text {abs }}$ (in minutes), extracted from the absorption curves of figure 12 .

crystallisation of the originally amorphous material, as observed in the progressive appearance of the peak corresponding to the lamellar stacking, and the Bragg reflections of the unit cell. In this case water absorption, until saturation, occurs for $\tau_{\text {abs }} \approx 500$ s, i.e. within the time $t_{0}$ over which no significant changes of the parameters of the lamellar structure are observed. The effective diffusion coefficient for water absorption is $D_{\text {wat }}^{6 \mathrm{I}} \approx 1.33 \times 10^{-10} \mathrm{~m}^{2} / \mathrm{s}$, which is comparable to the values obtained for PA6,6 and PA6,6/6T. The absorption kinetics are in very good agreement with those in Figure 10. After initial saturation, the amount of water starts to decrease. This is interpreted as an effect of crystallisation, in that the water is mainly contained within the amorphous phase and therefore with increase of crystallinity the saturation level decreases and water is expelled from the material. Thus, the overshoot in the sorption curves in Figure 12(a) is a further proof that solvent is effectively expelled from crystallites. Assuming that the solvent is fully expelled from crystallites and that absorption is unaffected by crystallization, the height of the overshoot might be used to estimate the crystallinity, which should then be of order $15 \%$ for both water and ethanol (Figure 12a). As indicated in figure 12a, water is expelled on a timescale which corresponds well to the characteristic time $\tau_{2}$ obtained from the evolution of the structural parameters of the lamellar stacking, indicating that this timescale is the one characteristic of the crystallisation process induced by the presence of water. Similarly, ethanol is expelled over a timescale which is compatible with that over which significant changes of the scattering curves are observed $\left(\tau_{1}^{\text {eth }}\right.$, reported in Figure $\left.12(\mathrm{a})\right)$. Note also that different from the 
already semi-crystalline polymers, the initial absorption of ethanol in PA6,6/6I is apparently compatible with Fickian diffusion, with a diffusion coefficient $D_{\text {eth }}^{6 \mathrm{I}} \approx 1.11 \times 10^{-9} \mathrm{~m}^{2} / \mathrm{s}$ which is considerably larger than for water.

\section{Temperature effects}

Absorption of water or ethanol into PA6,6/6I induces crystallisation, an effect which could be associated to the ability of solvents to rearrange the H-bonded structure of the system, allowing it to reach the thermodynamically stable state. It could also be related to the plasticization effect observed as a reduction of the global $T_{g}$ of the material, i.e. to a higher chain mobility which favors the building up of the crystalline arrangement. In the fully wet samples indeed the $T_{g}$ is $277 \mathrm{~K}$ and $260 \mathrm{~K}$ in water and ethanol respectively, which means that crystallisation is observed at $T-T_{g}=16 \mathrm{~K}$ and $33 \mathrm{~K}$, respectively. This latter effect should be also observed in the dry material when increasing $T$ above $T_{g}$.

We performed hence WAXS measurements in which the dry amorphous sample PA6,6/6I was kept at $T=399 \mathrm{~K}=T_{g}+36 \mathrm{~K}$ for 120 hours. Figure 13 shows WAXS spectra measured every 10 hours during this exposure. Some indications of local crystallisation are only visible at times longer than at least 2 days, in the appearance of a small peak corresponding to the 001 reflection at $Q=0.5 \AA^{-1}$ and eventually the indication of formation of a small shoulder for $1.6 \AA^{-1}<Q<1.7 \AA^{-1}$. These effects are still much less pronounced and even slower than changes induced by solvent absorption (Figure 4). This means that while enhancement of molecular mobility due to plasticization could be inducing some crystallisation in PA6,6/6I, the main driving force for this is the solvent absorption and possibly rearrangement of the H-bond structure.

This is also supported by neutron diffraction measurements of the effect of temperature 


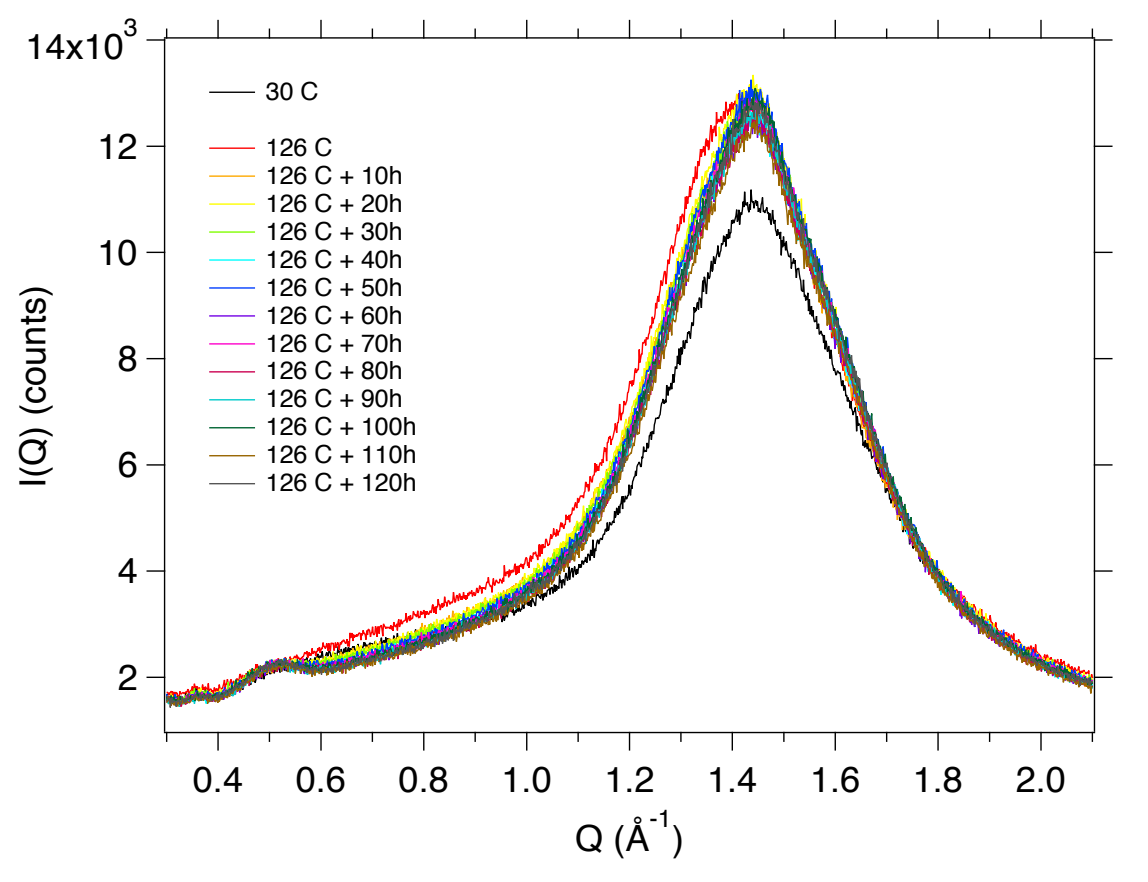

FIG. 13: WAXS spectra of PA6,6/6I at $T=399 K$, acquired at time steps of 10 hours, in the sequence indicated in legends, for a total measuring time of 120 hours.

on the crystallisation kinetics of PA6,6/6I in water. A fully wet sample (which just achieved solvent saturation) was measured at different increasing temperatures at intervals of approximately three hours. The maximum temperature was limited to $T=370 \mathrm{~K}$ in order to limit solvent evaporation during the measurements. During heating up, when the temperature reaches $T=330 \mathrm{~K}=T_{g}+53 \mathrm{~K}$, signs of crystallization at the local scale are already visible after a few hours, as indicated by the appearance of two peaks corresponding to the 100 and 010, 110 bragg reflections (Figure 14a). The peaks become even slightly more pronounced at $T=370 K=T_{g}+93 \mathrm{~K}$. The formed crystalline arrangement persists when the sample is cooled down to $T=300 K$ (Figure 14b). Crystallisation kinetics are therefore strongly accelerated with increasing temperature in wet samples, in comparison to the dry state (Figure 13) and the wet state at room temperature (Figure 10). 

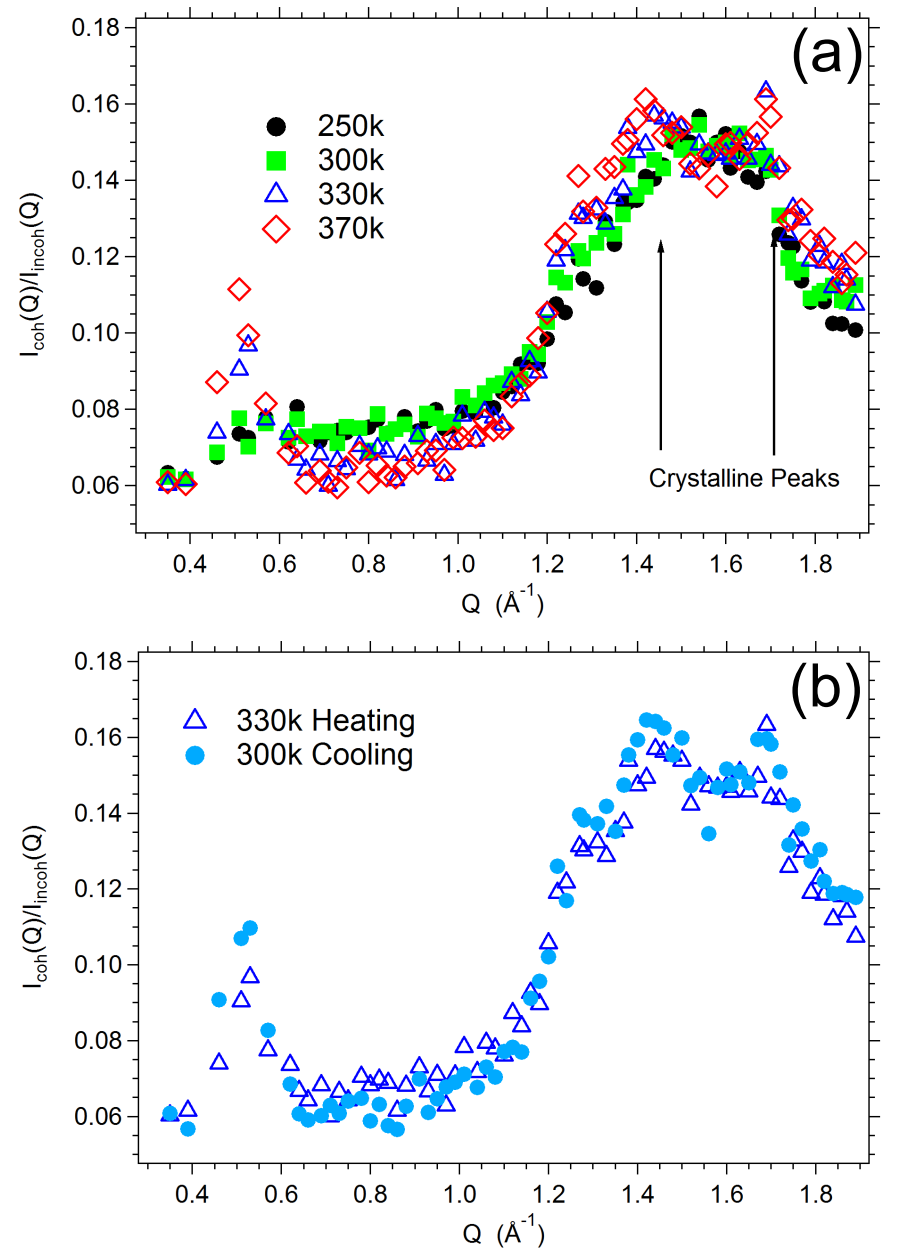

FIG. 14: (a) Neutron diffraction spectra for PA6,6/6I in in the wet state, for different increasing temperatures, as indicated. (b) Comparison of the spectra measured during heating up at $T=$ $330 \mathrm{~K}$ and after cooling down at $T=300 \mathrm{~K}$, as indicated.

\section{E. Proposed Scenario}

Different effects of the absorption of polar solvents on originally amorphous and semicrystalline polyamides were observed. In the semi-crystalline materials, the effects of polar solvent on the hydrogen-bond structure established in the dry state are small. Namely, a slight modification of the local crystalline organisation is observed. This effect could be related to the penetration of a limited amount of solvent molecules, with a very slow diffusion time, in the crystallites, which would induce some reorganization of hydrogen bonds in the 
crystal structure, allowing for the evolution towards a more stable structure (a structure with a higher index of crystalline perfection). Note though that additionally, reorganization of hydrogen bonds (or partial rotational mobility of hydrogen bonds, that is, of amide groups) in the crystallites may be driven by the increased mobility of the amorphous segments in the interlamellar regions, at crystalline layer boundaries. The change from $\alpha_{I}$ to $\alpha_{I I}$ phase at the Brill transition shows indeed that this is possible. However, we have shown that in the case of amorphous polymer, the solvent effect is not limited to plasticisation, i.e. an increase of the mobility in the amorphous phase. Moreover, the phenomena observed here take place at a temperature much lower than the Brill transition temperature. Therefore, it is likely that a combination of both processes is at play. In the originally amorphous material (PA6,6/6I) on the other hand the reorganisation of the hydrogen-bond structure is apparently much more significant and leads to crystallisation. This indicates that the original distribution of H-bonds in the material, which corresponds to the amorphous state, is possibly a nonequilibrium configuration: The solvent may then release some conformational constraints on segments, thus facilitating the path towards thermodynamic equilibrium and the formation of a regular network of hydrogen bonds which leads to partial crystallization. The enhanced mobility of the chains due to plasticisation possibly plays also a role, but it is not the exclusive reason for the onset of crystallisation, as shown by our investigation of temperature effects.

\section{CONCLUSIONS}

We reported results on the effects of water and ethanol absorption on the microscopic structure of PA6,6 and of amorphous (PA6,6/6I) and semi-crystalline (PA6,6/6T) semiaromatic random copolyamides. The most prominent structural modification induced by solvent absorption is crystallisation at room temperature of the originally amorphous PA6,6/6I 
copolymer, which is observed as the appearance of Bragg reflections in wide-angle scattering and of a pronounced peak characteristic of lamellar stacking in small angle X-ray scattering. The obtained final crystalline phase of PA6,6/6I resembles that of its semi-crystalline analogue and of PA6,6, but presents indications of some local higher degree of disorder. Kinetic measurements show that the development of the lamellar stacking is very slow and largely exceeds the characteristic time of the absorption kinetics. Moreover, the induced crystallisation is not, at least not entirely, an effect of plasticization, since annealing the dry sample at the same $T^{\mathrm{dry}}-T_{g}^{\mathrm{dry}}=T^{\mathrm{wet}}-T_{g}^{\text {wet }}$ induces only a much smaller degree of crystallinity over comparable kinetics times. Therefore the driving mechanism for the observed ordering could be the ability of polar solvents to break hydrogen bonds, together with the higher mobility due to plasticization. Note that the induced crystalline structure remains after drying, indicating a possible mechanism of tuning the crystallinity of PA6,6/6I by control of the slow crystallisation kinetics in the presence of water.

In the semi-crystalline copolymer PA6,6/6T, a swelling of the spacing of the lamellar stacking is observed during solvent absorption, as already observed for PA6,6 (also confirmed here) $[20,28]$ and PA6 [27]. This is attributed to the penetration of water into the amorphous regions in between the lamellae, as also supported by the fact that the kinetics of swelling correspond well to the absorption kinetics. The process observed in PA6,6/6T is therefore very different from that observed for PA6,6/6I and does not involve significant changes of the lamellar structure beyond the swelling effect described above. Note that this difference is particularly evident in the comparison of the structural kinetics observed for water and ethanol in the two copolymers: Whilst in PA6,6/6I the kinetics show faster variations for ethanol than for water, possibly associated with a stronger ability of rearranging the $\mathrm{H}$ bond structure and a more pronounced plasticization, in PA6,6/6T conversely are slower, 
according to the slower absorption of ethanol due to anomalous diffusion. Interestingly, even though the lamellar stacking only shows swelling effects and no hints of significant increase of crystallinity, we find evidences that the unit cell crystalline structure of PA6,6 and particularly PA6,6/6T, is changed in presence of water, tending to the more stable $\alpha_{I}$ phase. This might be at leat in part an effect of the penetration of a limited number of solvent molecules in the crystallites, which induces a partial reorganisation of the hydrogen bond structure.

\section{ACKNOWLEDGEMENTS}

M. L. acknowledges funding from the Softcomp network of excellence, Contract No. NMP3-CT-2004-502235 and from CNRS. A.A. acknowledges financial support from the projects MAT2012-31088 (Spain) and IT-654-13 (GV, Spain). This work is based on experiments performed at the DNS instrument operated by JCNS at the ForschungsNeutronenquelle Heinz Maier-Leibnitz (FRM II), Garching, Germany, and has been supported by the European Commission under the 7th Framework Programme through the 'Research Infrastructures' action of the 'Capacities' Programme, NMI3-II Grant Number 283883. We thank Y. Xu for assistance during the experiments at DNS.

[1] M. I. Kohan, ed., Nylon Plastics Handbook (Hanser: New York, 1995).

[2] S. M. Aharoni, n-Nylons: Their Synthesis, Structure, and Properties (Wiley: New York, 1997).

[3] H. K. Reimschuessel, J. Polym. Sci. Part D: Macromol. Rev. 12, 1977 (1985).

[4] D. R. Subramanian, A. Venkataraman, and N. V. Bhat, J. Macromol. Sci. Phys. 18, 177 
(1980).

[5] N. S. Murthy, J. Polym. Sci., Part B: Polym. Phys. 44, 1763 (2006).

[6] J. H. Magill, J. Polym. Sci. Part A-2 Polymer Physics 4, 243 (1966).

[7] F. Khoury, J. Polym. Sci. 33, 389 (1958).

[8] J. Mann and L. Roldan-Gonzalez, J. Polym. Sci. 60, 1 (1962).

[9] A. J. Lovinger, J. Appl. Phys. 49, 5003 (1978).

[10] A. J. Lovinger, J. Appl. Phys. 49, 5014 (1978).

[11] H. Miura, J. Hirschinger, and A. D. English, Macromolecules 23, 2169 (1990).

[12] N. G. McCrum, B. E. Read, and G. Williams, Anelastic and dielectric effects in polymer solids (John Wiley: New York, 1967).

[13] H. W. Starkweather, Water in Polymers, vol. 127 (ACS Symposium Series; American Chemical Society: Washington DC, 1980).

[14] E. Laredo and M. C. Hernandez, J. Polym. Sci., Part B: Polym. Phys. 35, 2879 (1997).

[15] E. Laredo, M. Grimau, F. Sánchez, and A. Bello, Macromolecules 36, 9840 (2003).

[16] K. Pathmanathan, J. Y. Cavaillé, and G. P. Johari, J. Polym. Sci., Part B: Polym. Phys. 30, 341 (1992).

[17] R. M. Neagu, E. Neagu, A. Kyritsis, and P. Pissis, J. Phys. D: Appl. Phys. 33, 1921 (2000).

[18] H. M. L. Huy and J. Rault, Polymer 35, 457 (1994).

[19] A. Koshimo, J. Appl. Polym. Sci. 9, 81 (1965).

[20] N. S. Murthy, Z. G. Wang, and B. S. Hsiao, Macromolecules 32, 5594 (1999).

[21] H. K. Reimschuessel, J. Polym. Sci. Polym. Phys. 16, 1229 (1978).

[22] X. Jin, T. S. Ellis, and F. E. Karasz, J. Polym. Sci., Polym. Phys. 22, 1701 (1984).

[23] M. Laurati, P. Sotta, D. R. Long, L.-A. Fillot, A. Arbe, A. Alegra, J. P. Embs, T. Unruh, 
G. J. Schneider, and J. Colmenero, Macromolecules 45, 1676 (2012).

[24] J. Pleštil, J. Baldrian, Y. M. Ostanevich, and V. Y. Bezzabotnov, Journal of Polymer Science 44, 449 (1991).

[25] H. Chen and P. Cebe, J. Therm. Anal. Calorim. 89, 417 (2007).

[26] I. Kolesov and R. Androsch, Polymer 53, 4770 (2012).

[27] N. S. Murthy, M. Stamm, J. P. Sibilia, and S. Krimm, Macromolecules 22, 1261 (1989).

[28] N. S. Murthy, M. K. Akkapeddi, and W. J. Orts, Macromolecules 31, 142 (1998).

[29] S. Rastogi, A. E. Terry, and E. Vinken, Macromolecules 37, 8825 (2004).

[30] E. Vinken, A. E. Terry, O. van Hasselen, A. B. Spoelstra, R. Graf, and S. Rastogi, Langmuir 24, 6313 (2008).

[31] J. A. W. Harings, Y. S. Deshmukh, M. R. Hansen, R. Graf, and S. Rastogi, Macromolecules 45, $5789(2012)$.

[32] E. Vinken, A. E. Terry, S. Hoffmann, B. Vanhaecht, C. E. Koning, and S. Rastogi, Macromolecules 39, 2546 (2006).

[33] N. S. Murphy, Macromolecules 20, 309 (1987).

[34] Y. S. Deshmukh, R. Graf, M. R. Hansen, and S. Rastogi, Macromolecules 46, 7086 (2013).

[35] C. W. Bunn and E. V. Garner, Proc. Roy. Soc. London A 189, 39 (1947).

[36] H. W. Starkweather and G. A. Jones, J. Polym. Sci., Polym. Phys. Ed. 19, 467 (1981).

[37] M. L. Colclough and R. Baker, J. Mat. Sci. 13, 2531 (1978).

[38] T. Itoh, Jpn. J. Appl. Phys. 15, 2295 (1976).

[39] J. Hirschinger, H. Miura, K. H. Gardner, and A. G. English, Macromolecules 23, 2153 (1990).

[40] Y. Lu, Y. Zhang, G. Zhang, M. Yang, S. Yan, and D. Shen, Polymer 45, 8999 (2004).

[41] L. Quintanilla, J. C. Rodríguez-Cabello, and J. M. Pastor, Polymer 35, 2321 (1994). 
[42] L. T. Lim, I. J. Britt, and M. A. Tung, J. Appl. Polym. Sci. 71, 197 (1999).

[43] O. B. Edgar and R. Hill, J. Polym. Sci. 8, 1 (1952).

[44] A. J. Yu and R. D. Evans, J. Polym. Sci. 42, 249 (1960).

[45] E. D. Harvey and F. J. Hybart, Polymer 12, 711 (1971).

[46] R. J. Gaymans, S. Aalto, and F. H. J. Maurer, J. Polym. Sci., Part A: Polym. Chem. 27, 423 (1989).

[47] A. Guinier, X-Ray Diffraction in Crystals, Imperfect Crystals and Amorphous Bodies (Dover Publications Inc., New York, 1994).

[48] F. Cser, J. Appl. Polym. Sci. 80, 2300 (2001).

[49] F. Alvarez, J. Colmenero, R. Zorn, L. Willner, and D. Richter, Macromolecules 36, 238 (2003).

[50] M. Laurati, P. Sotta, D. Long, A. Arbe, and J. Colmenero, in preparation (2013). 
Graphical Abstract: Effect of polar solvents on the crystalline phase of polyamides.

M. Laurati, A. Arbe, A. Rios de Anda, L.-A. Fillot and P. Sotta
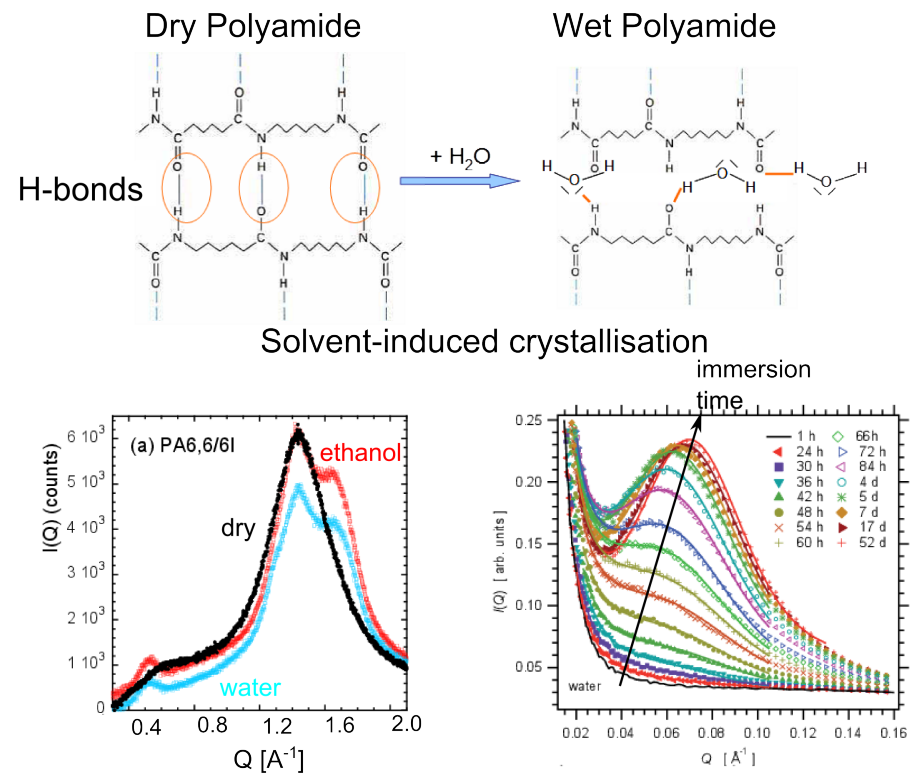

Structure: Unit Cell

Structure: Lamellar Stacking 